\title{
Photometric and morphological analysis of UCM galaxies. II. Statistical results and interpretation ${ }^{\star}$
}

\author{
A.G. Vitores ${ }^{1,2}$, J. Zamorano ${ }^{1}$, M. Rego ${ }^{1}$, J. Gallego $^{1}$ and O. Alonso ${ }^{1}$ \\ 1 Dpto Astrofísica, Facultad CC Físicas Universidad Complutense, E-28040 Madrid, Spain \\ 2 EUIT Industrial, Universidad Politécnica, E-28012 Madrid, Spain
}

Received July 31, 1995; accepted May 2, 1996

\begin{abstract}
We present the results of Thuan \& Gunn $r$ CCD imaging observations of the Universidad Complutense de Madrid (UCM) sample of emission-line galaxies (ELGs) selected by the presence of $\mathrm{H} \alpha$ emission in low resolution objective-prism spectra. In this work, a total of 212 objects from the UCM survey have been photometrically and morphologically characterized. In a previous paper, the observations, basic reductions and morphological classification for the UCM objects were presented. Here, we analyse the statistical properties of the sample and their interpretations. The main results obtained are as follows: (1) Our survey is specially effective in the detection of ELGs smaller and less luminous than normal spirals (the median values of the UCM sample are $M_{r}=-20.6$ and $D_{24}=19.4 \mathrm{kpc}$, for $H_{0}=50 \mathrm{~km} \mathrm{~s}^{-1} \mathrm{Mpc}^{-1}$ ). (2) The sample presents a very heterogeneous morphological population of galaxies. There is a clear predominance of late-type galaxies, with $66 \%$ being Sb or later. (3) We have found a set of galaxies specially interesting. In particular, we propose 9 candidates to BCD (blue compact dwarf galaxy), five of them previously unknown. (4) About $10 \%$ of the ELGs of the sample show typical parameters of S0 types, and in the subsample of 16 UCM interacting galaxies, $25 \%$ are S0 type galaxies. (5) In the set of UCM galaxies inside the Coma cluster, $71 \%$ exhibit Hubble types $\mathrm{Sb}$ or later.
\end{abstract}

Key words: surveys — galaxies: fundamental parameters - galaxies: starbust - galaxies: photometry

\section{Introduction}

The main aims of the UCM H $\alpha$ objective-prism survey are the identification and study of new young, low metallicity galaxies, together with the quantification of the properties of the star formation in the local universe. Details of the UCM survey have already been presented in the list I (Zamorano et al. 1994) and list II (Zamorano et al. 1996) papers. In order to achieve these goals, we have characterized photometrically (Vitores et al. 1996, Paper I of this series) and spectroscopically (Gallego et al. 1996) the UCM sample. From the set of parameters obtained (mainly through surface photometry techniques) in Paper I, in this work we analyse statistically the photometric properties of the UCM sample, and compare the results with those of other samples of galaxies.

In Sect. 2 we describe the statistical properties of the global photometric parameters, i.e. apparent and absolute magnitudes. In Sect. 3 we analyse the bulge+disk

Send offprint requests to: J. Zamorano

*Based on observations collected at the German-Spanish Astronomical Center, Calar Alto, Spain, operated by the MaxPlanck-Institut für Astronomie (MPIA), Heidelberg, jointly with the Spanish National Commision for Astronomy

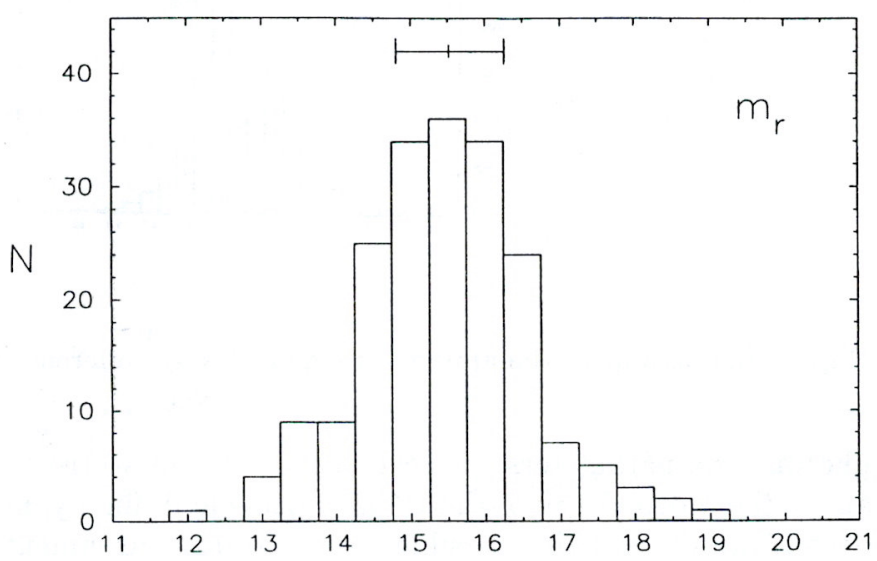

Fig. 1. Frequency histogram of the apparent magnitudes for the UCM sample. In this and the following histograms, the median and quartile points of each distribution are marked

$(B+D)$ decomposition parameters, whereas in Sect. 4 results about geometric parameters, mean photometric parameters and concentration indices are presented. Sect. 5 presents correlations and trends among several 

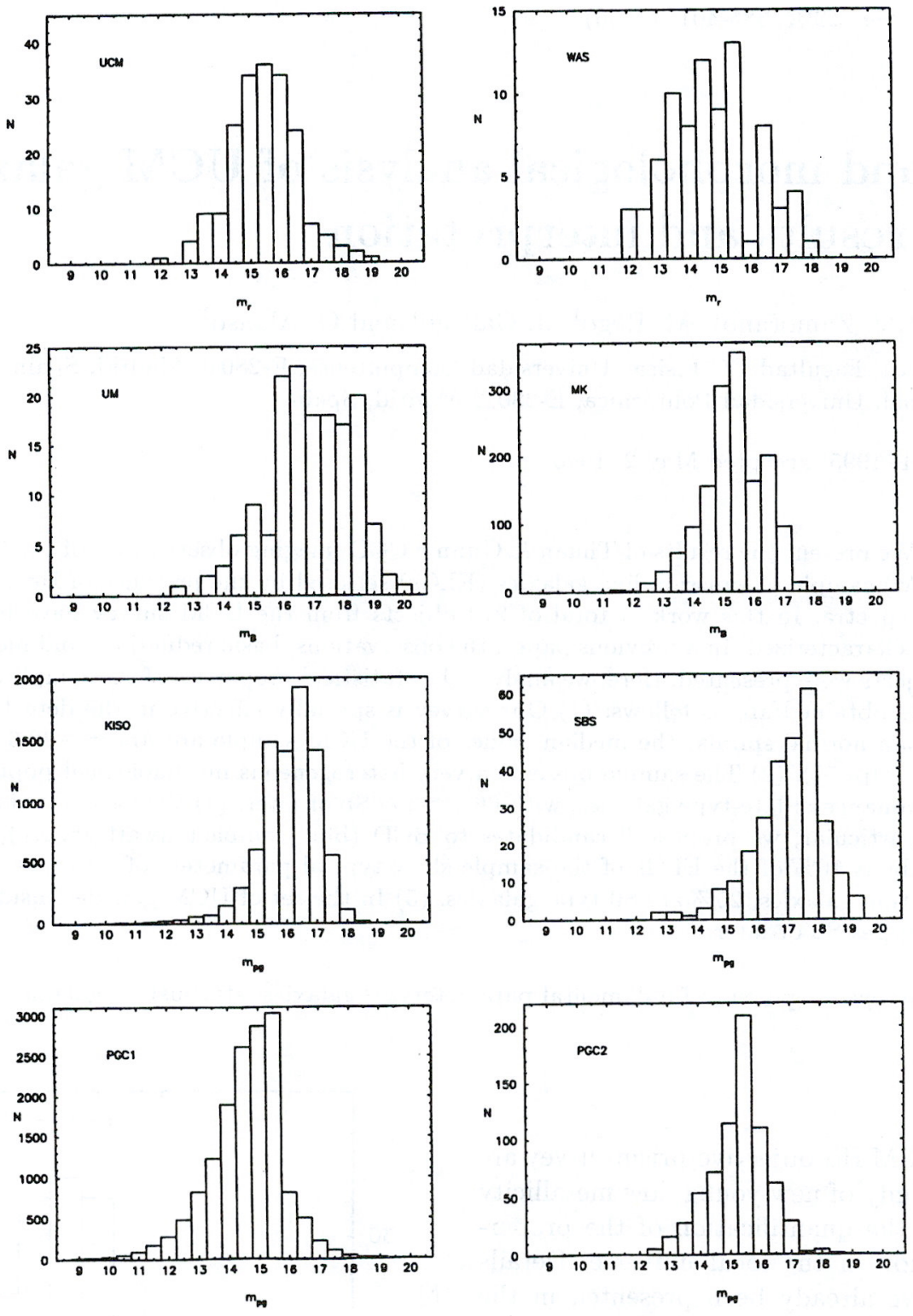

Fig. 2. Histograms of the apparent magnitudes for different samples of galaxies

photometric parameters. In Sect. 6 we perform statistics about the morphology of the UCM objects and, finally, in Sect. 7 we discuss the possible influence of environment on the presence and nature of ELGs.

\section{Global photometric parameters}

\subsection{Distribution of apparent magnitudes}

Figure 1 shows the frequency histogram of apparent magnitudes for the UCM objects in the $r$ filter of Thuan \& Gunn (1976), $m_{r}$, binned in 0.5 mag intervals. The distribution looks symmetrical around a central peak in the 15.5-16.0 interval and the mean value is 15.4. There is a paucity of objects brighter than $m_{r}=13$ since their spectra appear overexposed in the objective-prism plates, with the exception of galaxies with external HII regions of high contrast. There is also a low fequency of objects fainter than $m_{r}=18$, due to their low apparent luminosity (unless they present a high $\mathrm{H} \alpha$ emission flux). It is important to note that our sample is not apparent magnitudelimited. However, the instrumental objective-prism setup limits the detection to $z \leq 0.045$ (Gallego et al. 1996).

In order to show the influence of the different selection techniques, we compare in Fig. 2 histograms for different samples of galaxies. Wasilewski (WAS) sample (Wasilewski 1983), analysed in the $r$ band by Bothun et al. (1989), presents peaks at $m_{r} \sim 14.3$ and 15.3 (after 
transforming theirs $m_{24}$ to total $m_{r}$ using $m_{r}=m_{24}-0.21$ according to Kent 1984), and a mean and median values of $m_{r}=14.5$, that is, in average the UCM exploration is detecting objects $\sim 1 \mathrm{mag}$ fainter than those of the WAS survey.

The distribution of the University of Michigan (UM) survey in the blue (Salzer et al. 1989) is dominated by galaxies in the range $m_{B}=16.0-18.5$, with a mean value of $m_{B}=16.8 \pm 1.4$ and a median of $m_{B}=16.9$, which translates to $m_{r}=15.9$ assuming $\left(m_{B}-m_{r}\right) \simeq 1$ (Kent 1984 and Bothun et al. 1989). Thus the UM survey in the blue, with a higher redshift cutoff, is biased toward fainter objects, detecting galaxies about $0.4 \mathrm{mag}$ (in the median value) fainter than those of the UCM exploration in the red spectral range.

The distribution of the Markarian (MK) exploration (Mazzarella \& Balzano 1986), based on a color selection technique (UV excess), shows a more symmetrical shape than that of the UM survey, peaking at $m_{B}=15.5\left(m_{r}\right.$ $\simeq 14.5$ ) and showing a low detection of objects fainter than $m_{B}=17.0\left(m_{r} \simeq 16.0\right)$. The UCM exploration, like the UM survey, gets $\sim 2$ mag deeper than the MK sample. The comparison with the Kiso survey (Takase \& Miyauchi-Isobe 1993 and references therein) is difficult due to the uncertainties for $m_{\mathrm{pg}} \geq 16$. Mean magnitude for the Kiso survey is $m_{B}=16.1$, showing a tail toward brighter objects. The fraction of objects fainter than $m_{B}$ $=17.75\left(m_{r} \simeq 16.75\right)$ is only $1.5 \%$, whereas this fraction is $11.3 \%$ in the UCM sample.

The Second Byurakan Spectral Sky Survey (SBS) is an objective-prism exploration which makes use simultaneously of three colors. The available apparent magnitude histogram available for this survey is restricted to a subsample of blue compact galaxies (Izotov et al. 1993). This distribution peaks at $m_{B} \sim 17.7$ and gets, like the UM survey, deeper than the MK exploration. However, the bias of the SBS subsample toward faint galaxies prevent us from further comparisons.

Finally, we have constructed two histograms for normal galaxies using the PGC (Principal Galaxies Catalogue) of Paturel et al. (1989). The histogram named PGC1 corresponds to a subsample of 15330 galaxies with known apparent magnitudes and radial velocities. In comparison with our survey, the PGC1 sample shows a more prominent tail toward brighter objects with a lower fraction of galaxies fainter than $m_{r} \sim 15.8$ (3\% in the PGC1 sample and $11 \%$ for the UCM sample). The PGC2 histogram corresponds to a subsample of 705 galaxies selected from the PGC and laying on the UCM exploration fields. When comparing with the UCM sample this distribution is biased (by about $2 \mathrm{mag}$ ) toward brighter galaxies.

In summary, the UCM survey is biased against the detection of usually known, bright objects, and it is able to detect fainter objects (by $1-2 \mathrm{mag}$ ) than those of color

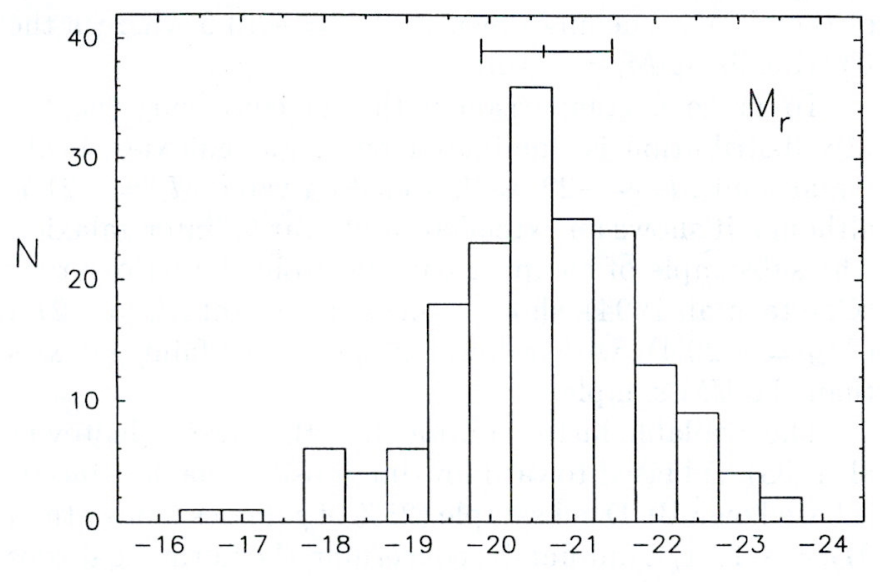

Fig. 3. Histogram of the absolute magnitudes for the UCM sample

surveys. With respect to the blue objective-prism surveys, the UCM exploration selects objects slightly brighter (by 0.4 mag.) than those of the UM survey.

\subsection{Distribution of the absolute magnitudes}

Absolute magnitudes, $M_{r}$, for the objects of the sample have been computed using the radial velocities obtained in the spectroscopic study of Gallego et al. (1996) which uses $H_{0}=50 \mathrm{~km} \mathrm{~s}^{-1} \mathrm{Mpc}^{-1}$. In Fig. 3 we show the histogram of the $M_{r}$ distribution for the UCM sample binned in 0.5 mag intervals. It looks rather symmetrical, peaking at $M_{r}=-20.5$. Absolute magnitudes span from $M_{r}=-23.5$ (for the luminous spiral UCM $2317+2356=$ NGC 7620 and the $\mathrm{cD} \mathrm{UCM} 2321+1631=$ NGC 7647) to $M_{r}=-16.7$ (for UCM 1612+1308 and UCM 2325+1628, candidates to BCD galaxies). The median of the distribution is -20.65 and the mean magnitude is $M_{r}=$ $-20.6 \pm 1.2$. Considering that a normal spiral has typically $M_{r}=-22.0$ (Bothun et al. 1989) or $M_{r}=-22.5$ (using $M_{B}=-21.5$ from Simien \& de Vaucouleurs (1986) and $B-r \simeq 1$ ), the UCM survey is detecting, in the mean, galaxies about $2 \mathrm{mag}$ fainter than normal spirals, in agreement with the findings of other ELGs explorations like WAS (Bothun et al. 1989) and UM (Salzer et al. 1989). This confirms the advantage of objective-prism techniques for detecting intrinsically faint objects.

In Fig. 4 we compare the absolute magnitudes histograms for different samples of galaxies (transforming their values to $H_{0}=50 \mathrm{~km} \mathrm{~s}^{-1} \mathrm{Mpc}^{-1}$ scale). In the WAS sample, analyzed by Bothun et al. (1989), the distribution is more irregular than that of the UCM sample, due to the relatively low number of galaxies (74). The median value of the WAS sample is -20.3 . Concerning the UM sample, the median absolute magnitude is $M_{r}=-20.0\left(M_{B}=\right.$ $-19.0)$, i.e. $\sim 0.7 \mathrm{mag}$ fainter than the $\mathrm{UCM}$ value. The UM survey spans a wider range in luminosities than that 
of the UCM, reaching down to $M_{r}=-13.6$ whereas the $\mathrm{UCM}$ ends at $M_{r}=-16.7$.

To make a comparison with the color surveys, the MK distribution is dominated by bright galaxies, peaking around $M_{r} \sim-22$, with a median value $M_{r}=-21.7$, although it shows an extended tail toward fainter galaxies. The subsample of compact galaxies from the Kiso survey (Comte et al. 1994), shows a median value of $M_{r}=-21.4$ $\left(M_{B}=-20.4\right)$, with a lower frequency of faint galaxies than the UM sample.

The available histogram for the SBS survey (Izotov et al. 1993), is biased toward low-luminosity galaxies since it is based on a BCD subsample (25\% of galaxies fainter than $\left.M_{B}=-17.4\right)$. And finally, concerning the normal galaxies catalogues (PGC1 and PGC2, see above), they both peak at $M_{r}=-22\left(M_{B}=-21\right)$, i.e. $\sim 1.5$ mag brighter than the UCM ELGs sample.

To summarize, surveys based on the presence of emission-lines shows median values of $M_{r}$ about $1 \mathrm{mag}$ fainter than the samples of galaxies selected by color or the general catalogues. In a relative context, although the UCM exploration presents a contribution of only $8 \%$ at the faint range $M_{r}>-19.0$ (compare with the $31 \%$ of the UM blue survey), this contribution is more important than the $2 \%$ obtained in the PGC2 subsample.

\section{Photometric parameters of the bulge + disk decomposition}

The bulge+disk $(B+D)$ decomposition of the surface brightness profile for the UCM sample has yield a set of objects (6\% of the sample) which do not allow this technique of analysis (see Paper I). However, additional information obtained from the spectroscopic study (Gallego et al. 1996) has shown that all of them are field stars. Thus, in this section we analyse the results from this decomposition technique only for the remaining objects.

\subsection{Distribution of the $B / D$ ratios}

In Fig. 5, we show the histogram of bulge-to-disk luminosity ratios $(B / D)$ for the UCM survey. As it can be seen, the UCM sample is dominated by low values of $B / D: 70 \%$ of the galaxies with $B / D \leq 1$ (and $60 \%$ with $B / D \leq 0.75$ ).

From the correlation found by Kent (1985) in the $r$ bandpass between $B / D$ and $T$ (Hubble type), it follows that the UCM sample is dominated by late-type spirals $(T \geq \mathrm{Sb})$, although the $B / D$ ratio is only one of the five morphological parameters used in this work (see Paper I). Moreover, one should be aware of the low reliability of the decomposition for galaxies whith $B / D \geq 1.70$ (i.e. $B / T \geq$ $0.63)$. Nevertheless, this is not an important effect in our sample since only $18 \%$ of the UCM galaxies exceeds this $B / D$ threshold. When excluding these cases, the mean $B / D$ for the sample is 0.5 , a typical value for Sb type galaxies (Kent 1985).

\subsection{Distribution of bulge parameters}

Figures 6 and 7 represent the histograms for the parameters of the bulge component obtained from the $B+D$ decomposition. In Fig. 6 it is shown that the distribution of the effective bulge surface brightness, $\mu_{\mathrm{e}}$, is dominated by faint bulge components, as it should be expected in a late-type dominated sample of galaxies. The mean $\mu_{\mathrm{e}}$ is $22.5 \pm 1.6 \mathrm{mag} / \operatorname{arcsec}^{2}$ and when excluding the cases with $B / D \geq 1.70$, this value remains almost unalterable $\left(22.7 \pm 1.6 \mathrm{mag} / \operatorname{arcsec}^{2}\right)$, indicating that neither the mean value nor the dispersion are an artifact caused by our $B+D$ decomposition technique. Since $\mu_{\mathrm{e}} \simeq 22.0$ for $\mathrm{Sb}$ and $\simeq 23.0$ for Sc galaxies (Kent 1985; Simien 1989), it is shown again that the UCM ELGs sample is dominated by Sb-Sc types.

In Fig. 7 we show the histogram of the effective radius, $r_{\mathrm{e}}$ (containing one-half the total light of the bulge component). The sample is again dominated by short scale bulges, with a median value of $2.1 \mathrm{kpc}$. Only four galaxies exhibit $r_{\mathrm{e}}>9 \mathrm{kpc}$ (UCM 1314+2827, with an anomalous $B+D$ decomposition; UCM $2300+2014$, with a PSF correction probably overestimated due to a high seeing value; UCM $2320+2428$, with a difficult decomposition due to its high inclination, and UCM $2321+1631$, an early-type galaxy). Even taking into account the high dispersion in $r_{\mathrm{e}}$ for a given Hubble type (Kent 1985; Simien 1989), the UCM sample is dominated by late-type galaxies (consider that typically $r_{\mathrm{e}} \sim 2.5 \mathrm{kpc}$ for Sb-Sc). Finally, since the two bulge parameters result more affected by seeing, we must be especially careful when comparing the results between different authors.

\subsection{Distribution of disk parameters}

Figures 8 and 9 present the histograms for the parameters of the disk component obtained from the $B+D$ decomposition. Figure 8 shows the histogram of the disk (face-on) extrapolated central surface brightness, $\mu_{0}$. In contrast to the $\mu_{\mathrm{e}}$ histogram, the $\mu_{0}$ distribution is dominated by bright components, with median and mean values of $20.3 \mathrm{mag} / \operatorname{arcsec}^{2}$ (20.2 if we exclude the galaxies with $B / D \geq 1.70$ ). Moreover, $75 \%$ of the sample is concentrated within a narrow range of only $2 \mathrm{mag}$, in agreement with Kent (1985). There is also a wide range in $\mu_{0}$ which results narrowed when the cases with $B / D \geq 1.70$ are not considered. Thus, all of the UCM galaxies with $\mu_{0}$ $>23.25 \mathrm{mag} / \operatorname{arcsec}^{2}$ have $B / D>2.2$ and exhibit morphologic parameters more typical of S0 systems. In these galaxies, as Kent (1985) pointed out, the $B+D$ decomposition is quite unreliable since the reliability of the decomposition depends primarily on the accuracy of $r^{1 / 4}$ law in describing the bulge, which could produce a spurious disk component.

An important topic about the $\mu_{0}$ values is the existence of a universal $\mu_{0}=21.65 \pm 0.30 B$-mag/ $\operatorname{arcsec}^{2}$ as 


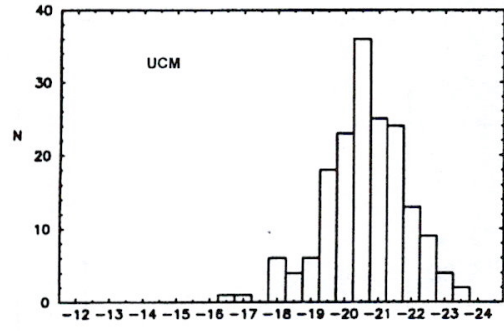

Mr
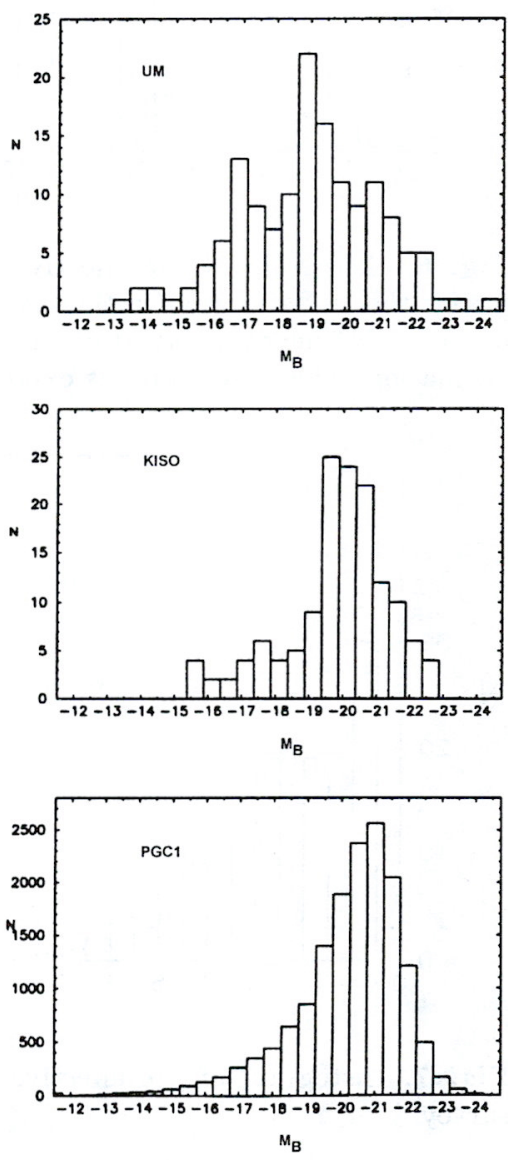

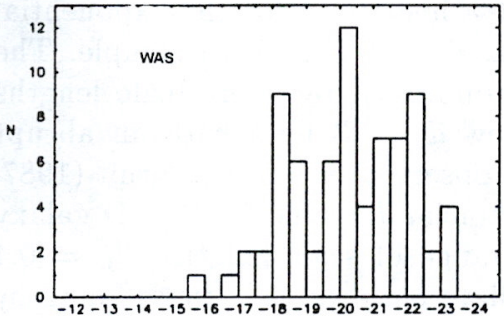

$\mathrm{Mr}_{\mathrm{r}}$
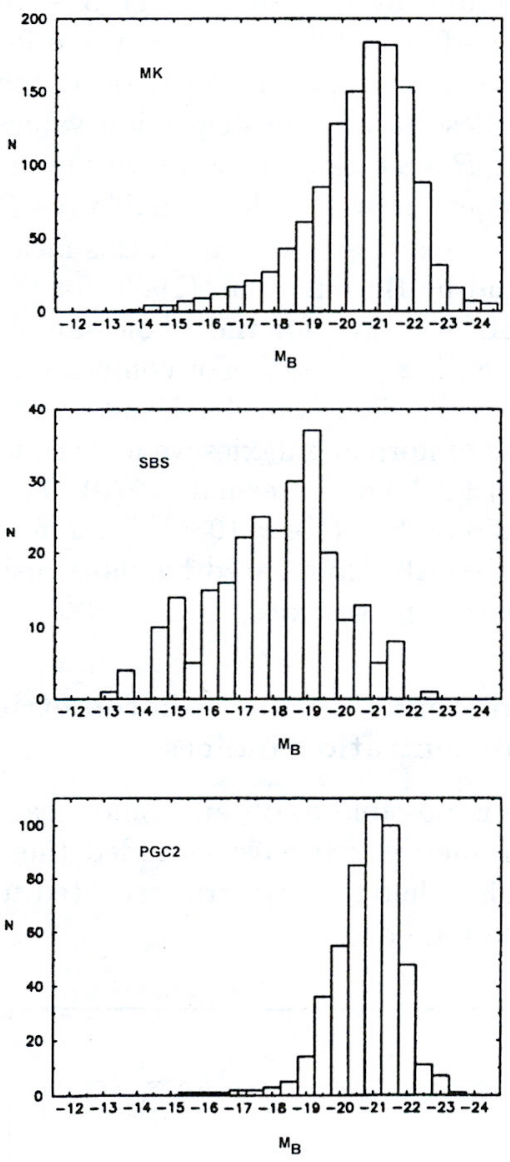

Fig. 4. Histograms of the absolute magnitudes for different samples of galaxies

proposed by Freeman (1970) and confirmed by Boroson (1981) and Simien \& de Vaucouleurs (1986). It must be noted, however, that Romanishin et al. (1983) find a mean $\bar{\mu}_{0}=22.74 B-\mathrm{mag} / \operatorname{arcsec}^{2}$ for low surface brightness spirals whilst van der Kruit (1987) finds a brighter value of $\bar{\mu}_{0}=22.5 B-\mathrm{mag} / \operatorname{arcsec}^{2}$ for spirals with disk length of only $\sim 1.5 \mathrm{kpc}$. For the UCM sample, the mean central surface brightness is $\bar{\mu}_{0}=21.3 \mathrm{~B}-\mathrm{mag} / \operatorname{arcsec}^{2}$ (assuming $B-r \simeq 1$ ), i.e. $0.35 \mathrm{mag} / \operatorname{arcsec}^{2}$ brighter than the Freeman value and $0.2 \mathrm{mag} / \operatorname{arcsec}^{2}$ brighter than the mean for the Kent (1985) sample. This result evidences that the disks of ELGs are slightly brighter than those of normal spirals due to the influence of the $\mathrm{H} \alpha$ emission (included in the $r$ bandpass). This effect, together with the trends found for ELGs to be usually smaller and less luminous than normal spirals, has been also observed by Bothun et al. (1989) for the Wasilewski sample. Although their mean value $\left(\mu_{0} \simeq 19.4 r-\mathrm{mag} / \operatorname{arcsec}^{2}\right)$, is about 1 mag brighter than ours, it must be noted that the bulge component is not considered in the analysis of the surface brightness profiles made by these authors, increasing then artificially the disk contribution. In our sample, we find a high dispersion (1.8 $r$-mag/ $\left.\operatorname{arcsec}^{2}\right)$ around the mean $\mu_{0}$ value, although this is reduced to $0.9 \mathrm{mag} / \operatorname{arcsec}^{2}$ if galaxies with a high $B / D$ ratio are excluded (see above). Thus, the final mean value for $\mu_{0}$ is $20.2 \pm 0.9 \mathrm{mag} / \operatorname{arcsec}^{2}$ for the set of UCM galaxies having a more reliable $B+D$ decomposition. 
In Fig. 9 we present a histogram for the exponential scale length of the disk, $d_{\mathrm{L}}$, for the UCM sample. The distribution is clearly dominated by short scale lengths ( $65 \%$ of the sample show $d_{\mathrm{L}}<3 \mathrm{kpc}$ ) with an abrupt fall for higher values, as observed by van der Kruit (1987, 1989). Excluding the artificial $d_{\mathrm{L}}$ value for the $\mathrm{cD}$ galaxy UCM $2321+1631$, our range of scale lengths $\left(d_{\mathrm{L}}=0.4\right.$ $-14.7 \mathrm{kpc})$ is intermediate between the ranges found by Freeman (1970), 1 - $12 \mathrm{kpc}$, or van der Kruit $(1987,1989)$, $1-11 \mathrm{kpc}$, and those found by Boroson (1981), $3-16$ $\mathrm{kpc}$, or Kent (1985), $1.5-0 \mathrm{kpc}$. The mean $d_{\mathrm{L}}$ value for the whole UCM sample is $3.2 \mathrm{kpc}$, although with a high dispersion of $2.8 \mathrm{kpc}$. These mean and dispersion values are not produced by our $B+D$ decomposition technique since they remain unchanged even if the less reliable $B+D$ decomposition galaxies are excluded. Moreover, this mean $d_{\mathrm{L}}$ is similar to that found by Bothun et al. (1989) for the Wasilewski sample of ELGs (4 kpc for the whole sample and $3.2 \mathrm{kpc}$ for galaxies with $z<0.033)$. For comparison, our Galaxy presents $d_{\mathrm{L}}=7.5 \mathrm{kpc}$ (van der Kruit 1989), and from several samples of normal galaxies we have computed a mean $d_{\mathrm{L}}=4.1 \pm 2.6 \mathrm{kpc}$ (Freeman 1970), $7 \pm 3$ $\mathrm{kpc}$ (Boroson 1981) and $6 \pm 4 \mathrm{kpc}$ (Kent 1985). Therefore the UCM sample is statistically dominated by short disk scales, more typical of late-type galaxies.

\section{Geometric parameters, mean photometric parameters and concentration indices}

Since even for the stars is possible to obtain some meaningless photometric parameters, we have excluded these objects from any analysis. Therefore, we are restricted to a set of 194 galaxies (see Paper I).

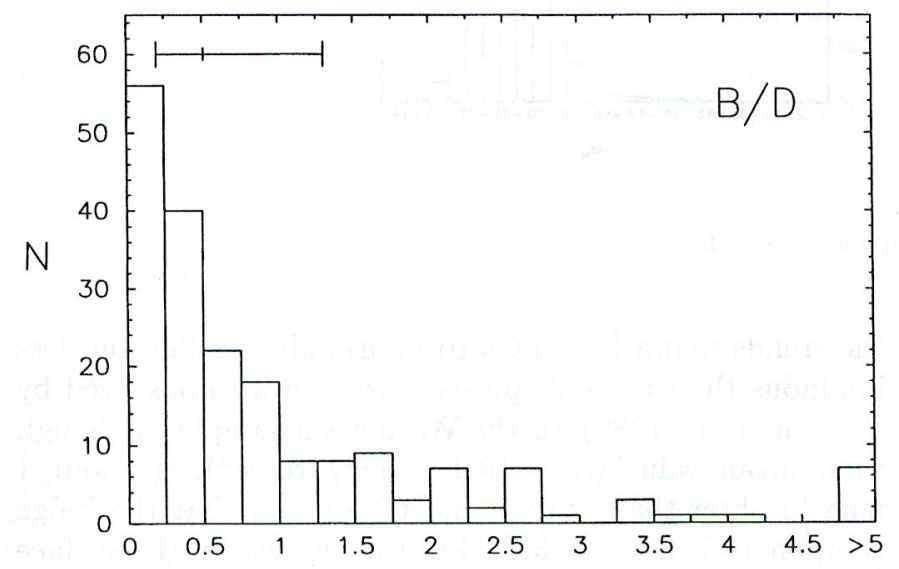

Fig. 5. Histogram of the $B / D$ ratios for the UCM survey

\subsection{Geometric parameters}

Figure 10 presents a histogram of the diameters, measured at the $24 \mathrm{mag} / \operatorname{arcsec}^{2}$ level, for the UCM galaxies. There is

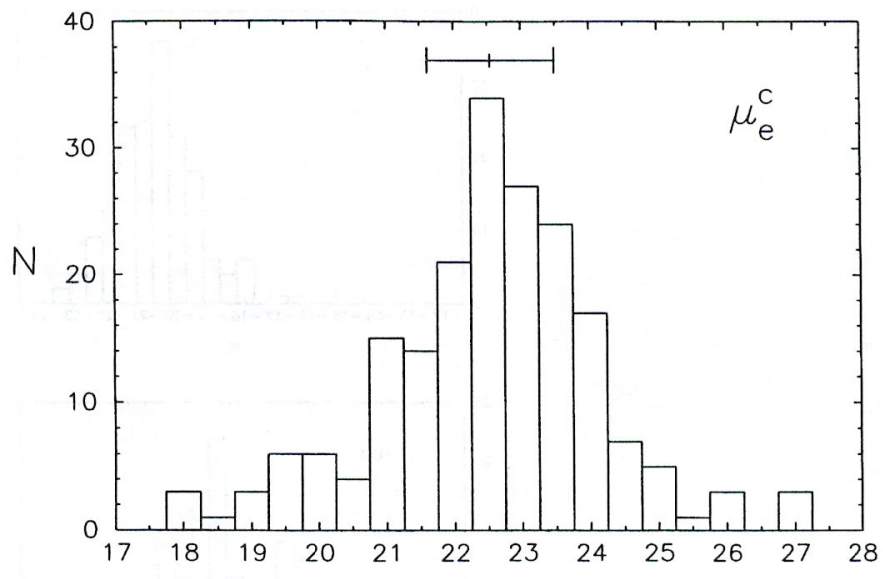

Fig. 6. Histogram of the effective bulge surface brightness, $\mu_{\mathrm{e}}$, for the UCM survey (in this and the following figures, superindex " $c$ " denotes corrected for galactic extinction and for inclination, when necessary, as exposed in Paper I)

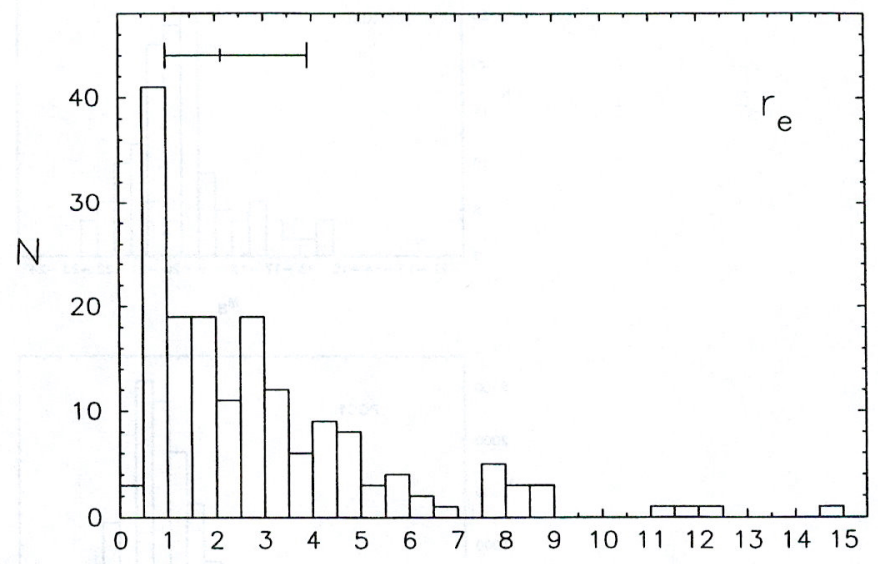

Fig. 7. Histogram of the effective radius, $r_{\mathrm{e}}$, for the UCM survey

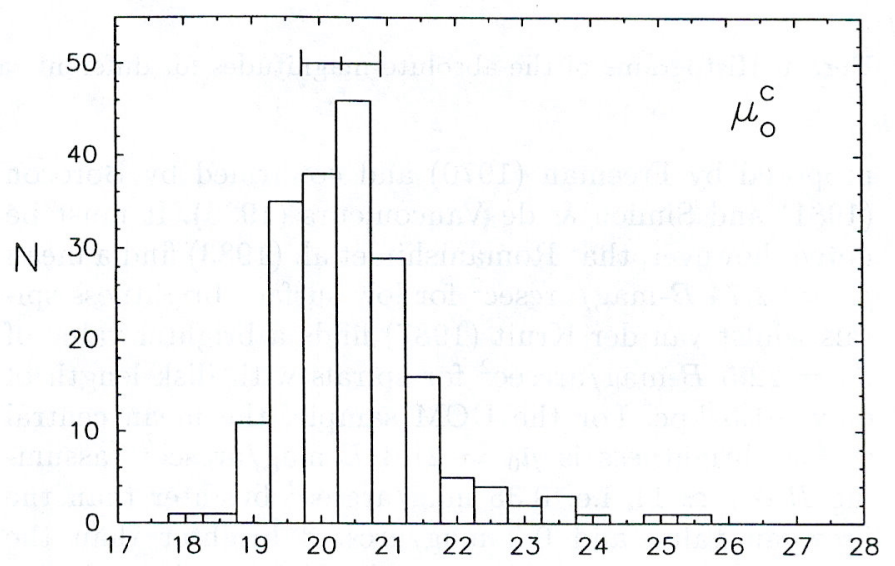

Fig. 8. Histogram of the disk face-on extrapolated central surface brightness, $\mu_{0}$ for the UCM survey 


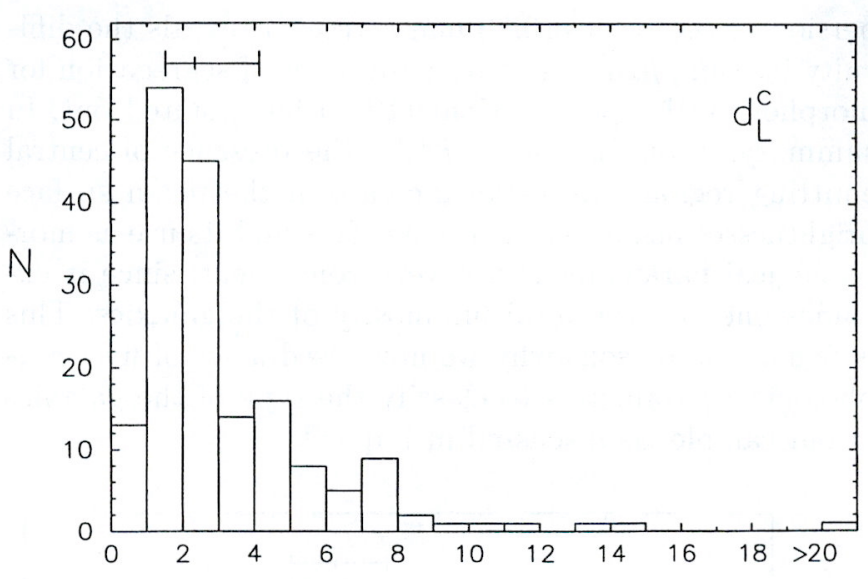

Fig. 9. Histogram of the exponential scale length of the disk, $d_{\mathrm{L}}$, for the UCM survey

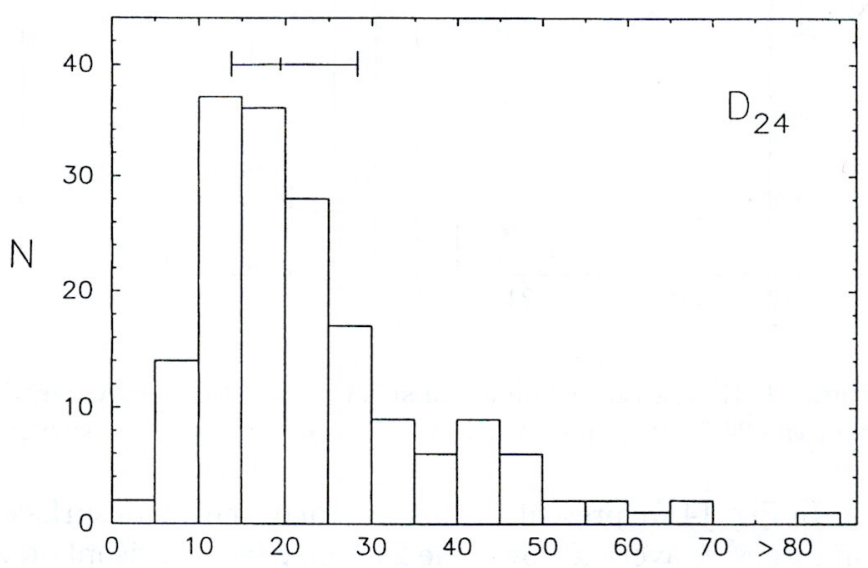

Fig. 10. Histogram of the diameters, measured at the 24 $\mathrm{mag} / \operatorname{arcsec}^{2}$ level, for the UCM survey

a clear predominance of the small sizes, with $78 \%$ of galaxies with $D_{24}<30 \mathrm{kpc}$ (nearly the size of our Galaxy). This is an expected result since the objective-prism technique favours the detection of smaller and less luminous systems in which emission features appear more contrasted, as Bothun et al. (1989) pointed out in their study of the Wasilewski survey. Our sample covers a wide range of sizes (3-67 kpc, excluding the cD UCM 2321+1631), the median is $19.4 \mathrm{kpc}$ and the mean value is $23 \pm 14 \mathrm{kpc}$.

For comparison, the Wasilewski sample (Bothun et al. 1989) ranges up to $90 \mathrm{kpc}$, being dominated by the smallest galaxies. Although their fraction of galaxies larger than $30 \mathrm{kpc}$ is $36 \%$ (higher than in our sample), their whole sample has a mean value of $26 \mathrm{kpc}$, quite similar to our result. The available data for the University of Michigan survey (Salzer et al. 1989) have allowed us to compare our $D_{24}$ values in the r band with $D_{25}$ in the $B$ band. The UM sample extends up to $80 \mathrm{kpc}$ and exhibits also a predominance of small galaxies, with $29 \%$ having $D_{24}<7.5 \mathrm{kpc}$
( $5 \%$ in the UCM sample) and only $10 \%$ of galaxies larger than $30 \mathrm{kpc}$ ( $22 \%$ in the UCM sample). The mean diameter for the UM is $17 \mathrm{kpc}$, although with a large dispersion of $\pm 14 \mathrm{kpc}$, and with a median value of $14 \mathrm{kpc}$, i.e. $\sim 30 \%$ smaller than in our sample. In summary, the UCM survey is detecting, in average, galaxies slightly smaller than the WAS survey but larger than the UM sample. Finally, it must be noted that size is one of the most important parameters to classify ELGs into different groups (Salzer et al. 1989). These authors find that all the galaxies with diameters above $22.5 \mathrm{kpc}$ are Sy or SBN (starburst nuclei) and, using a sample of 48 Sy from MacKenty (1990), we compute a mean $\bar{D}_{24}=25 \pm 10 \mathrm{kpc}$. In the UCM sample, we confirm the trend of these active galaxies to have larger sizes $\left(\bar{D}_{24}=36 \pm 15 \mathrm{kpc}\right.$ for our subset of Sy galaxies).

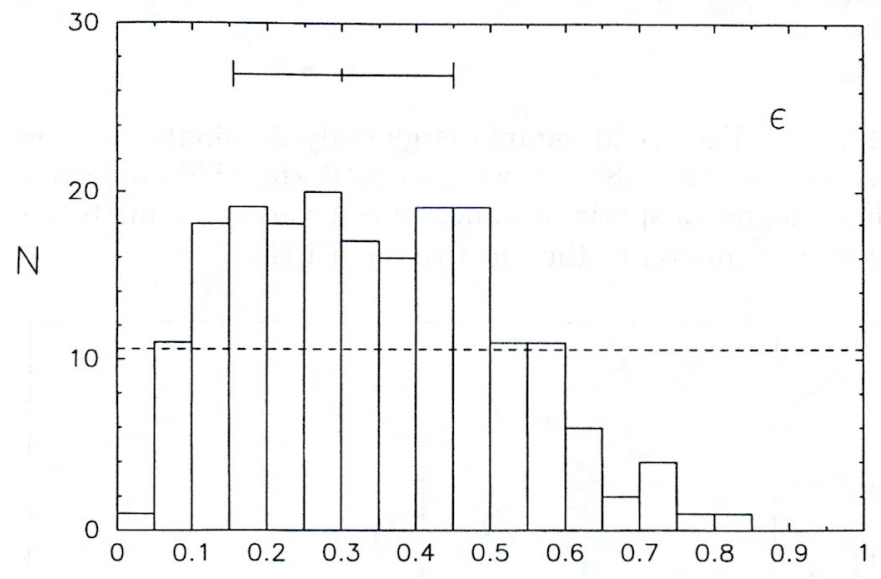

Fig. 11. Histogram of the observed ellipticity $\epsilon$ for the UCM survey. The dashed line indicates the frequency for randomly orientated disks

Another geometric parameter that we have calculated in our sample is the ellipticity $\epsilon$ (averaged over the five most external isophotes better defined). The distribution, shown in Fig. 11, reveals a nearly uniform distribution for $\epsilon<0.5$ and an steep decline of detections above this value. The distribution does not show the expected uniformity for a sample of disks randomly oriented, but is dominated by low-inclinations objects. Thus, only $17 \%$ have $\epsilon>0.5$, showing the difficulty in detecting nearly edge-on systems due to their higher internal extinction.

\subsection{Mean photometric parameters}

In Figs. 12 to 14 we present the histograms of the mean photometric parameters, calculated in Paper I. These parameters are important due to their independence on the decomposition procedure used in the analysis of the radial distribution of the light in the galaxies. Figure 12 shows the histogram of the effective radius, $a_{\mathrm{e}}$ (containing onehalf the total luminosity of a galaxy). The median is 2.8 $\mathrm{kpc}$ and the mean is $3.8 \mathrm{kpc}$, but with a dispersion of 


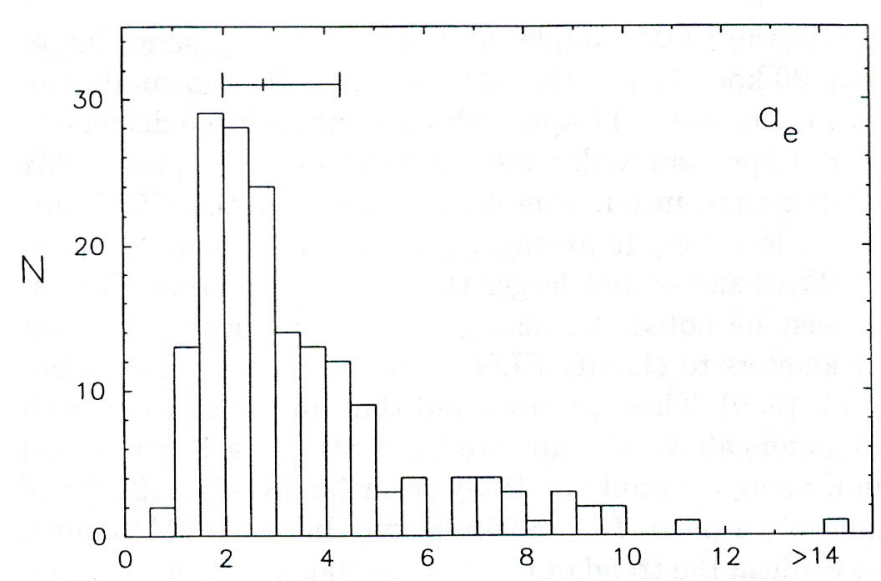

Fig. 12. Histogram of the effective radius, $a_{\mathrm{e}}$, for the UCM survey

$4.6 \mathrm{kpc}$. The UCM sample is globally dominated by low $a_{\mathrm{e}}$ values, with $88 \%$ showing $a_{\mathrm{e}} \leq 6 \mathrm{kpc}$. This reflects a high degree of spatial luminosity concentration due to the central emission in the most of our ELGs.

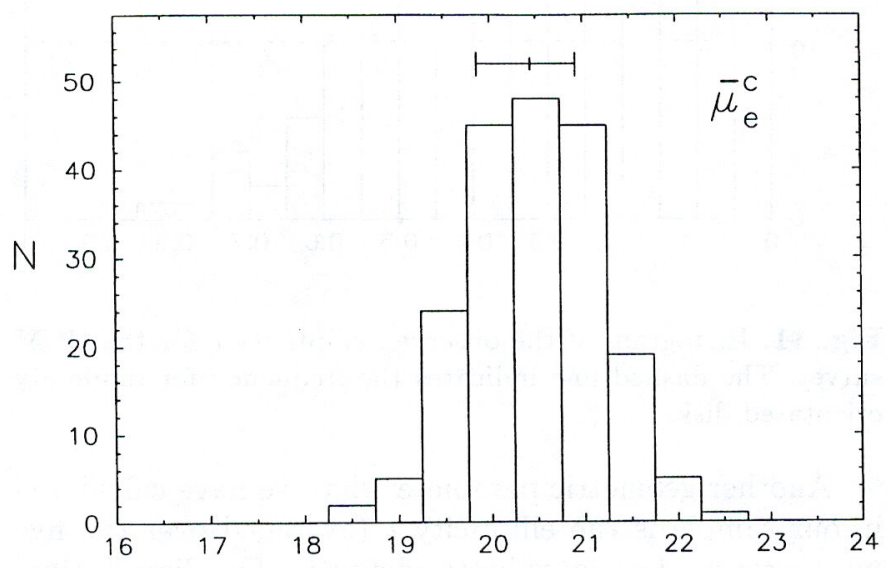

Fig. 13. Histogram of the mean surface brightness inside the effective radius, $\bar{\mu}_{\mathrm{e}}$, for the UCM survey

In Fig. 13, the distribution of mean surface brightness inside the effective radius, $\bar{\mu}_{\mathrm{e}}$, is shown. Although the histogram covers a range of about $4.5 \mathrm{mag}$, quite similar to that found by Kent (1985), most of the galaxies spans over a range of only $1.5 \mathrm{mag}\left(19.75-21.25 \mathrm{mag} / \mathrm{arcsec}^{2}\right)$ which includes $67 \%$ of the sample, and the mean value is $20.46 \pm 0.70 \mathrm{mag} / \mathrm{arcsec}^{2}$. In comparison, we have obtained from the Kent (1985) sample a mean value of $20.73 \pm 0.70$ $\mathrm{mag} / \operatorname{arcsec}^{2}$ for normal galaxies, whereas for the low surface brightness galaxies (LSB) from Romanishin et al. (1983) the mean is $\sim 22.6 \mathrm{mag} / \operatorname{arcsec}^{2}$ (using $B-r \simeq 1$ ). Therefore, on the one hand, the mean value of $\bar{\mu}_{\mathrm{e}}$ for the UCM sample is $\sim 0.3 \mathrm{mag}$ brighter than that of normal galaxies. And, on the other hand, the relatively low dis- persion of the values $\left(0.70 \mathrm{mag} / \operatorname{arcsec}^{2}\right)$ reveals the difficulty in using $\bar{\mu}_{\mathrm{e}}$ as the only parameter of segregation for morphological types, as Kent (1985) has pointed out. In summary, in our sample of ELGs the presence of central emitting regions causes an increase in the mean surface brightnesses inside the effective radius and its use as morphological parameter is not very convenient, since it excludes one-half the total luminosity of the galaxies. This is one of the reasons why we have used a set of five morphological parameters to classify the type of the galaxies in our sample, as discussed in Paper I.

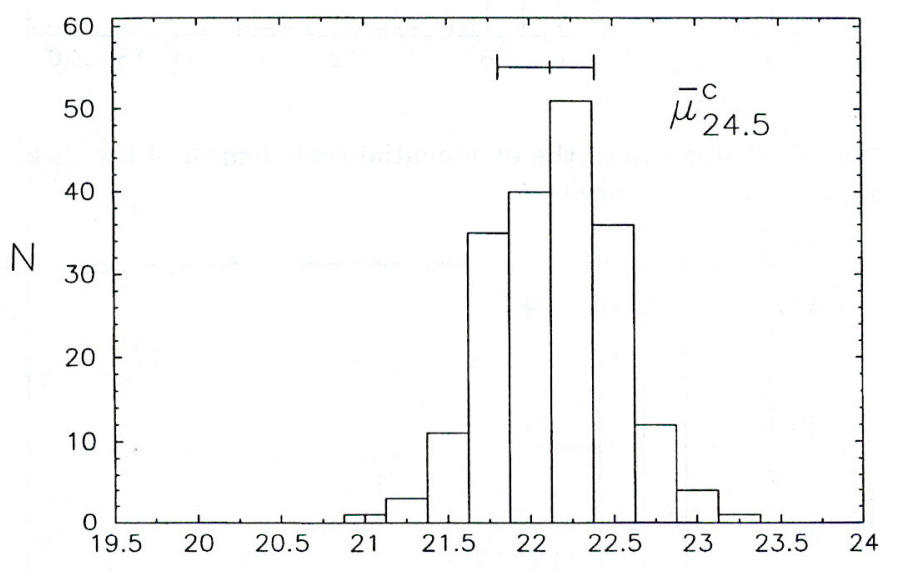

Fig. 14. Histogram of the mean surface brightnesses averaged over the $24.5 \mathrm{mag} / \operatorname{arcsec}^{2}$ isophote, $\bar{\mu}_{24.5}$, for the UCM survey

In Fig. 14 we present the histogram of the mean surface brightnesses averaged over the $24.5 \mathrm{mag} / \operatorname{arcsec}^{2}$ isophote. The range for ELGs spands only about $2.5 \mathrm{mag}$ and $78 \%$ of the sample is inside a narrow range of only $1 \mathrm{mag}(21.6-$ $22.6 \mathrm{mag} / \operatorname{arcsec}^{2}$ ). Considering the diversed morphology of the UCM galaxies, that result reflects the insufficiency of using a mean surface brightness as the only parameter for morphological segregation. The mean value is $22.14 \pm$ $0.36 \mathrm{mag} / \mathrm{arcsec}^{2}$, typical of normal galaxies (Doi et al. 1993; Okamura et al. 1994). Thus, whereas $\bar{\mu}_{\mathrm{e}}$ is quite sensitive to the presence of nuclear bursts, $\bar{\mu}_{24.5}$, averaged over almost the whole galaxy, is unaffected by the emission features. Moreover, the low dispersion on $\bar{\mu}_{24.5}$ indicates that this mean brightness can not be used as the only parameter for morphological segregation.

\subsection{Concentration indices}

Histograms for the three concentration indices used in Paper I are shown in Figs. 15-17. Figure 15 represents the distribution for $c_{\text {in }}(\alpha)$, with $\alpha=0.3$, a parameter introduced by Doi et al. (1993). The range is $0.24 \leq c_{\text {in }}(\alpha)$ $\leq 0.70$, similar to that found by Okamura et al. (1994) for normal galaxies in the $R$ band. The distribution is quite symmetrical around a median of 0.48 , and with $66 \%$ of the objects in the range $0.40-0.60$. The mean 


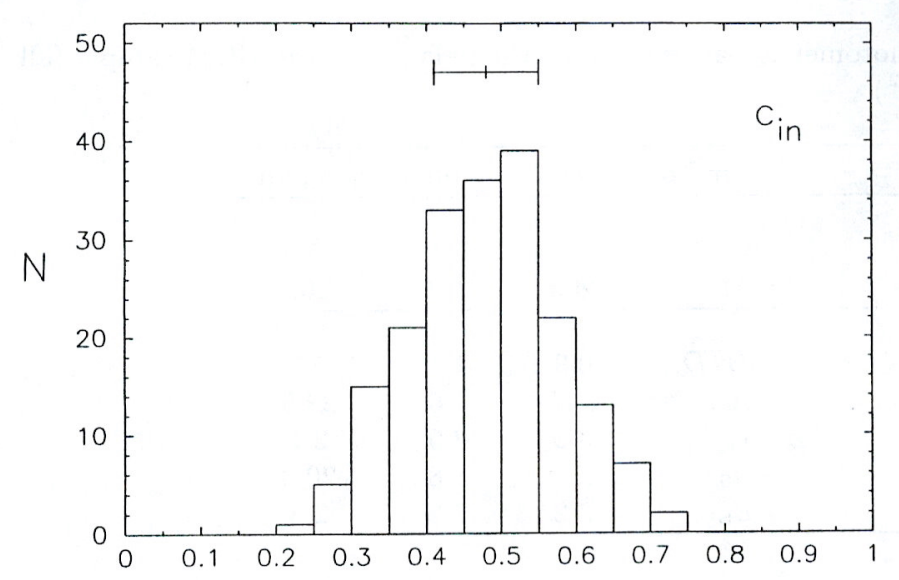

Fig. 15. Histogram for the $c_{\text {in }}(\alpha)$ values for the UCM survey

value for the UCM ELGs is $0.47 \pm 0.10$, a typical result for Hubble types later than Sa (Doi et al. 1993). Then, due to the insufficiency of this index as the only morphological parameter, we have used the biparametrical classification $\left(c_{\mathrm{in}}(\alpha), \bar{\mu}_{24.5}\right)$ proposed by these authors as one of the five morphological criteria (see Paper I).

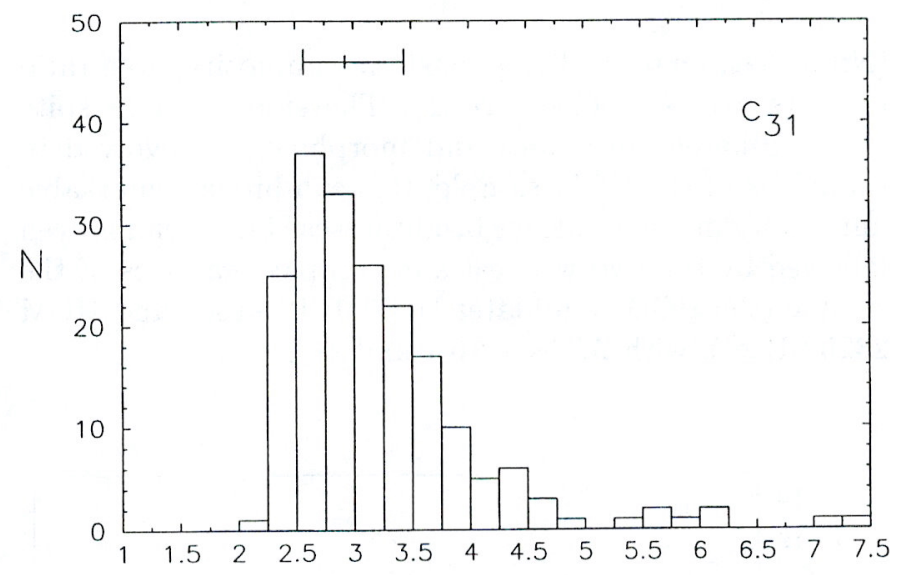

Fig. 16. Histogram for the values of the $c_{31}$ concentration index for the UCM survey

In Fig. 16 we present the histogram for the $c_{31}$ concentration index introduced by de Vaucouleurs (1977). The wide range reveals the diversity of morphological types in our ELG sample (2.8 is the theoretical value for a pure exponential profile and 7.0 for a pure $r^{1 / 4}$ law). The histogram is mainly populated by low values of $c_{31}$, as expected for a sample dominated by late-type galaxies. The median is 3.0 and the mean is $3.2 \pm 0.8$, a value corresponding to Hubble types later than Sa (Gavazzi et al. 1990), and $83 \%$ of the values fall between 2.25 and 3.75 . A steep decline is observed for $c_{31}<2.25$, a value only attained by some Irr in the sample of Gavazzi et al. (1990). In fact, in the UCM sample, only UCM 1303+2908 (an irregu- lar ELG in Coma) is below this value. The deficiency of galaxies with $c_{31}>4.5$ represents the paucity of Hubble types S0 or earlier in our sample. Moreover, $42 \%$ of the sample shows $c_{31}<2.8$ (the theoretical value for a pure disk model), a similar value to the $49 \%$ found from the sample of normal galaxies of Gavazzi et al. (1990). Concerning the Sy subsample, although some of them exhibit $c_{31}$ as high as 7.21, others have values of 2.65. Thus, as expected, this parameter does not represent any advantage in segregating active galaxies. Finally, for the UCM subsample of BCDs, we obtain for $c_{31}$ a mean of $2.6 \pm 0.3$, that is a very low value when comparing with the mean for the whole sample, suggesting a rather late Hubble type for this kind of galaxies.

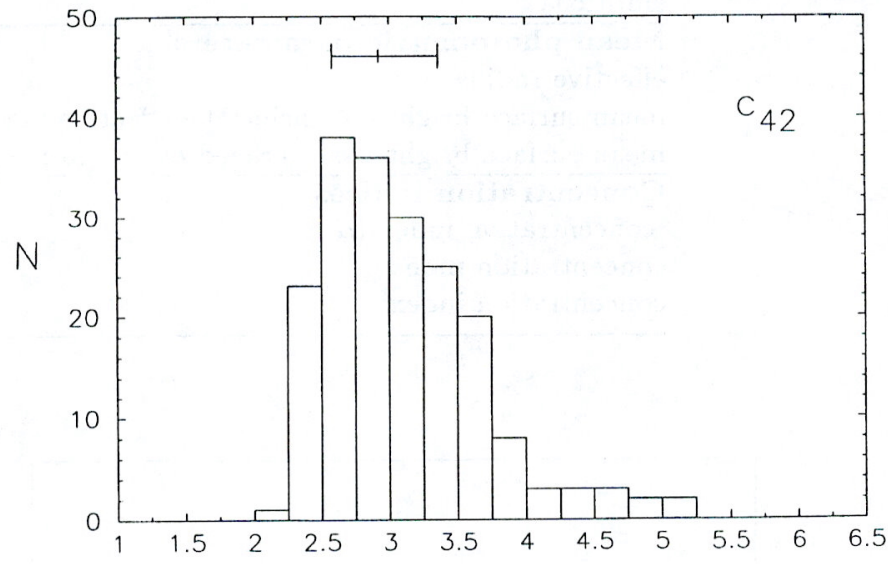

Fig. 17. Histogram for the values of the $c_{42}$ concentration index for the UCM survey

In Fig. 17 we show the histogram of the third concentration index used in this work, the $c_{42}$ parameter introduced by Kent (1985). The range is the expected for a sample with a varied morphology (the $c_{42}$ theoretical values are 2.74 for a pure exponential profile and 7.0 for a pure $r^{1 / 4}$ law). The distribution shows $78 \%$ of the values in the $2.25-3.75$ band, a typical range for Sb morphologies or later (Kent 1985). The median value is 3.0 and the mean is $3.1 \pm 0.6$, a typical value for $\mathrm{Sb}-\mathrm{Sc}^{+}$types. The relatively low fraction of galaxies with $c_{42}=4.50-$ 5.13 is dominated by galaxies that correspond to S0 types. And, finally, concerning the Sy and BCD subsamples, the results are the same as those for the $c_{31}$ index.

5. Correlations between the absolute, geometrical, mean photometric and concentration parameters

In the previous section we have presented the statistical results of the UCM sample. These results have been summarized in Table 1 for a quick look. Now, in this section we analyse some trends and correlations between the parameters discussed above. 
Table 1. Mean, standard deviation and median values of the photometric parameters for the galaxies in the UCM sample (all the scales are in $\mathrm{kpc}$ and the surface brightnesses in $\mathrm{mag} / \operatorname{arcsec}^{2}$ )

\begin{tabular}{|c|c|c|c|c|}
\hline & symbol & mean & st.dev. & median \\
\hline \multicolumn{5}{|l|}{ Magnitudes } \\
\hline apparent magnitude & $m_{r}$ & 15.4 & 1.1 & 15.4 \\
\hline absolute magnitude & $M_{r}$ & -20.6 & 1.2 & -20.7 \\
\hline \multicolumn{5}{|l|}{$B+D$ parameters } \\
\hline bulge to disk ratio & $B / D$ & 0.9 & 1.1 & 0.5 \\
\hline effective bulge surface brightness & $\mu_{\mathrm{e}}$ & 22.5 & 1.6 & 22.5 \\
\hline effective radius of the bulge & $r_{\mathrm{e}}$ & 3.0 & 3.0 & 2.1 \\
\hline disk face-on central surface brightness & $\mu_{0}$ & 20.3 & 1.8 & 20.3 \\
\hline exponential scale length of the disk & $d_{\mathrm{L}}$ & 3.2 & 2.8 & 2.3 \\
\hline \multicolumn{5}{|l|}{ Geometric parameters } \\
\hline diameters at $24 \mathrm{mag} / \operatorname{arcsec}^{2}$ & $D_{24}$ & 23.0 & 13.6 & 19.4 \\
\hline ellipticity & $\epsilon$ & 0.3 & 0.2 & 0.3 \\
\hline \multicolumn{5}{|l|}{ Mean photometric parameters } \\
\hline effective radius & $a_{\mathrm{e}}$ & 3.8 & 4.6 & 2.8 \\
\hline mean surface brightness inside the effective radius & $\bar{\mu}_{\mathbf{e}}$ & 20.5 & 0.7 & 20.5 \\
\hline mean surface brightness averaged over $24.5 \mathrm{mag} / \mathrm{arcsec}^{2}$ & $\bar{\mu}_{24.5}$ & 22.1 & 0.4 & 22.1 \\
\hline \multicolumn{5}{|l|}{ Concentration indices } \\
\hline concentration index $(\alpha=0.3)$ & $c_{\text {in }}(\alpha)$ & 0.5 & 0.1 & 0.5 \\
\hline concentration index & $c_{31}$ & 3.2 & 0.8 & 3.0 \\
\hline concentration index & $c_{42}$ & 3.1 & 0.6 & 3.0 \\
\hline
\end{tabular}

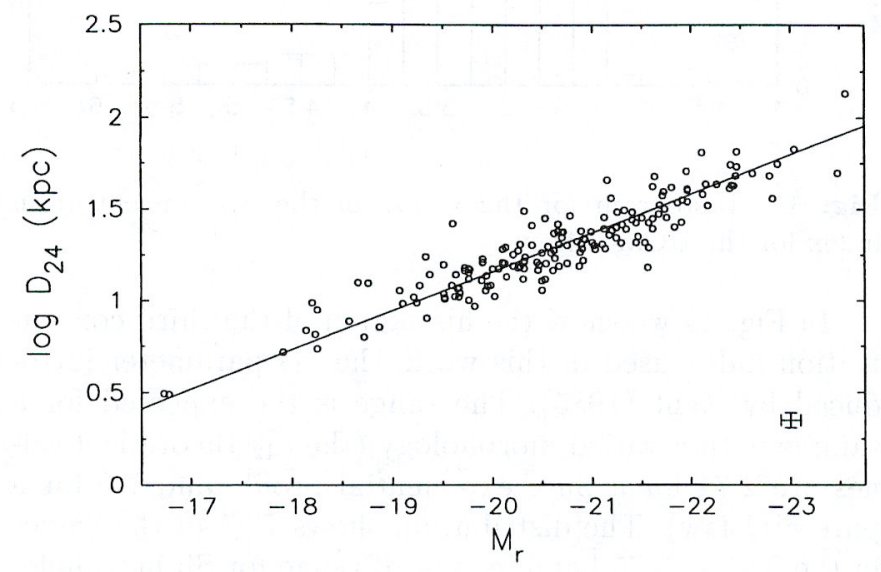

Fig. 18. Correlation between the diameter at the 24 mag/arcsec ${ }^{2}$ level and the absolute $r$ magnitude for the UCM survey. A linear least-squares fit to the data is shown by a straight line. In this and the following plots, typical error bars are marked

\subsection{Size-luminosity relation}

Figure 18 shows the tight correlation between the diameter at the $24 \mathrm{mag} / \operatorname{arcsec}^{2}$ level and the $r$ absolute magnitude for the UCM sample. A least-square fit to our data leads to:

$$
\log D_{24}=(-3.10 \pm 0.13)+(-0.21 \pm 0.01) M_{r}
$$

(Note that, analytically, a constant luminosity/area ratio predicts $\log D=C-0.20 M)$. Therefore, and in spite of the different processes and morphologies involved in the ELGs of the UCM sample, this exhibits a remarkable uniformity in mean surface brightnesses. This trend is even followed by the two weakest and compact galaxies of the sample (the BCD candidates UCM 1612+1308 and UCM $2325+1628$ ), with $M_{r} \sim-16.7$ and $D_{24} \sim 3 \mathrm{kpc}$.

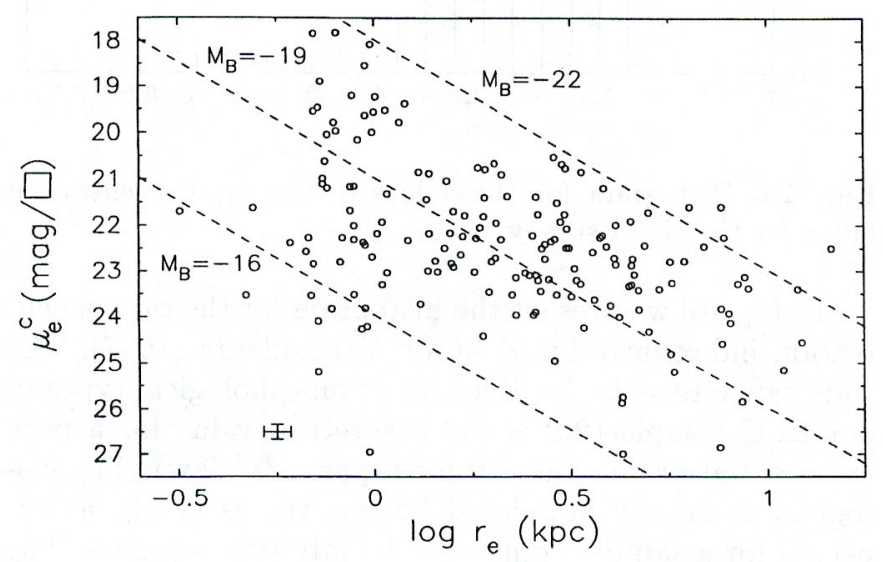

Fig. 19. $\mu_{\mathrm{e}}$ vs. $\log r_{\mathrm{e}}$ distribution for the bulge component of the UCM survey. Lines of constant absolute magnitude for bulges are indicated by dashed lines 


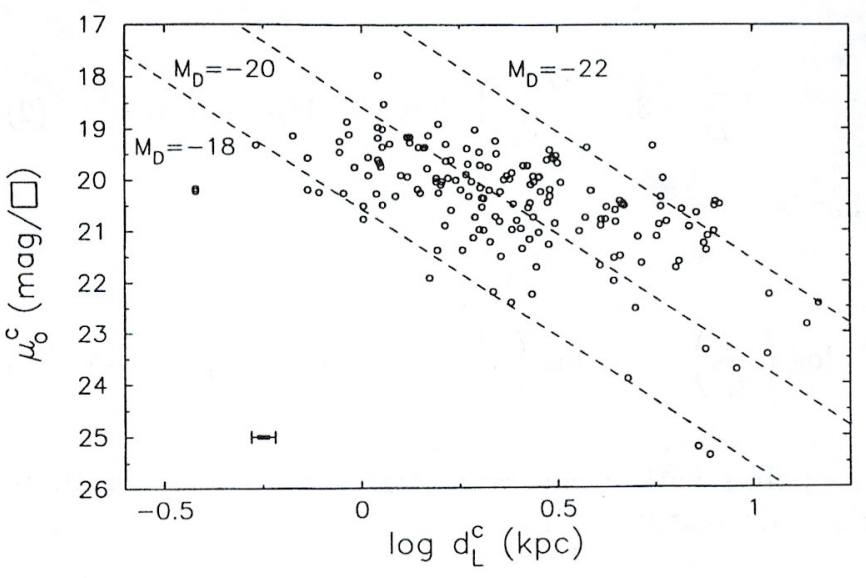

Fig. 20. $\mu_{0}$ vs. $\log d_{\mathrm{L}}$ distribution for the disk component of the UCM survey. Lines of constant absolute magnitude for disks are indicated by dashed lines

\subsection{Relations concerning $B+D$ parameters}

Figures 19 and 20 show the $\mu_{\mathrm{e}}$ vs. $\log r_{\mathrm{e}}$ distribution for the bulge component and the $\mu_{0}$ vs. $\log d_{\mathrm{L}}$ for the disk component, respectively. Concerning the bulge distribution, there is some evidence of a trend between radius and surface brightness. Comparing with the lines of constant luminosity $\left(M_{\text {Bulge }}=\right.$ constant corresponds to $\mu_{\mathrm{e}}=C+5$ $\log r_{\mathrm{e}}$ ), the large scatter of points indicates a wide range in absolute magnitudes. The same effect is observed by Kent (1985), Simien \& de Vaucouleurs (1986) and Kodaira et al. (1986), although the effect of the seeing correction on the bulge parameters prevent us from further comparisons. A similar trend between the disk parameters is observed in Fig. $20\left(M_{\text {Disk }}=\right.$ constant corresponds to $\mu_{0}=C+5 \log$ $\left.d_{\mathrm{L}}\right)$. In this case, the scatter is lower than that obtained for the bulges and it is similar to that found by Kodaira et al. (1986), although a bit larger than that observed by Simien \& de Vaucouleurs (1986). Moreover, this diagram shows that the majority of the disks in our sample exhibit disk luminosities weaker than those of normal late type spirals $\left(M_{\text {Disk }} \sim-22\right)$. Since $\mu_{0}$ value is (in the mean) slightly brighter than in normal spirals, that effect is explained as due to shorter disk length-scales in our ELGs.

Also in connection with the $B+D$ decomposition, we have investigated the trends between the four parameters derived from this technique and the bulge-to-total luminosity ratio, $B / T$, as proposed by Kent (1985). In principle, a drop in the $B / D$ ratio may be due to either a decrease in bulge size relative to the disk, a drop in bulge surface brightness, or a combination of both effects (Kent 1985). To investigate this, on the one hand, we plot in Fig. 21 the relation between the ratio of scale lengths and $B / T$. Although there is a trend in the sense that the scale lengths ratio increases with $B / T$ (especially if the less reliable cases, with $B / T \geq 0.63$, are excluded), the scatter on

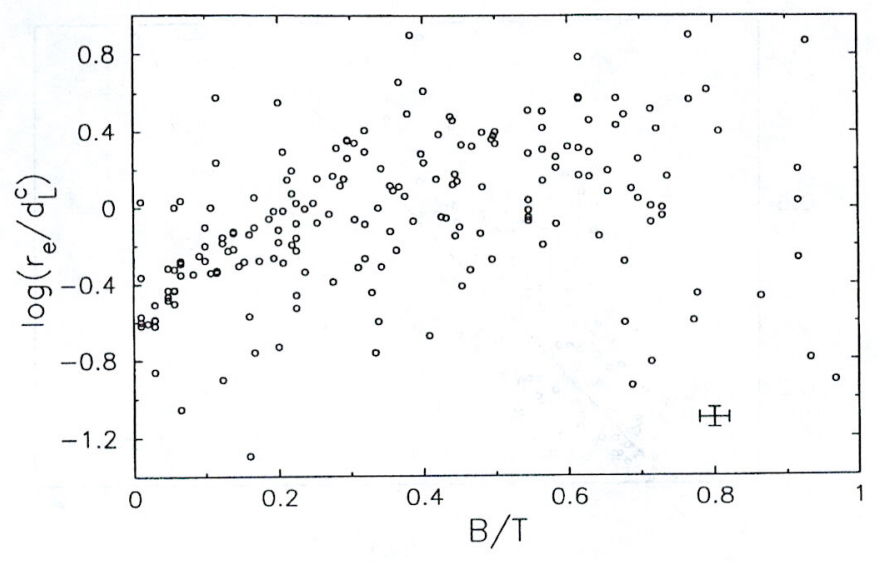

Fig. 21. Relation between the ratio of scale lengths and the $B / T$ ratio for the UCM survey

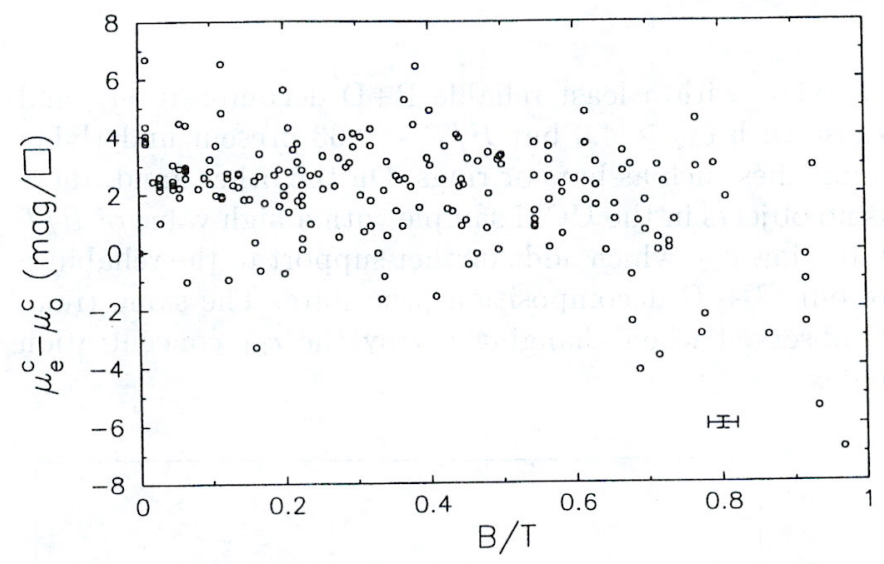

Fig. 22. Relation between the difference of surface brightnesses and the $B / T$ ratio for the UCM survey

the plot is remarkable. On the other hand, although this author proposes that a dimming of the bulge rather than a brightening of the disk would be primarily responsible for a drop in $B / T$, for our sample there is no relation between the difference of surface brightnesses and $B / T$ ratio (Fig. 22). However, the difficulty in unifying the different $B+D$ decomposition techniques could be responsible for the observed discrepancies among different authors.

Since the concentration indices are introduced due to their advantage in measuring the concentration of light in the galaxies with independence of any decomposition procedure, the relation between $B / T$ ratio and the concentration index $c_{42}$ has been investigated. In Fig. 23 we observe a trend in $B / T$ to increase with $c_{42}$, as found by Kent (1985). Only objects with $c_{42} \geq 4.0$ (corresponding to early type galaxies) deviate from the mean relation, but since $87 \%$ of the UCM sample has $c_{42}<3.75$, the contribution of those points is negligible. On the one hand, all the cases with $B / T>0.63$ and $c_{42} \geq 4.0$ correspond to S0 


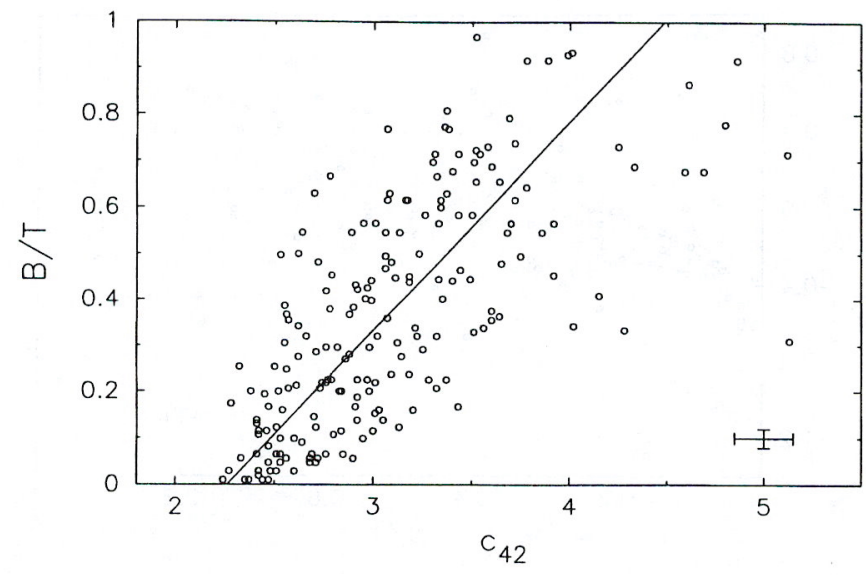

Fig. 23. Relation between the $B / T$ ratio and the concentration index $c_{42}$ for the UCM survey. A linear least-squares fit to the data is shown

galaxies (with a least reliable $\mathrm{B}+\mathrm{D}$ decomposition), and those with $c_{42} \geq 4.0$ but $B / T<0.63$ present underlying anomalies such as bars or rings. On the other hand, there is no objects in the UCM sample with a high value of $B / T$ but a low $c_{42}$, which adds further support to the reliability of our $B+D$ decomposition procedure. The same trend is observed when changing $c_{42}$ by the $c_{31}$ concentration index.

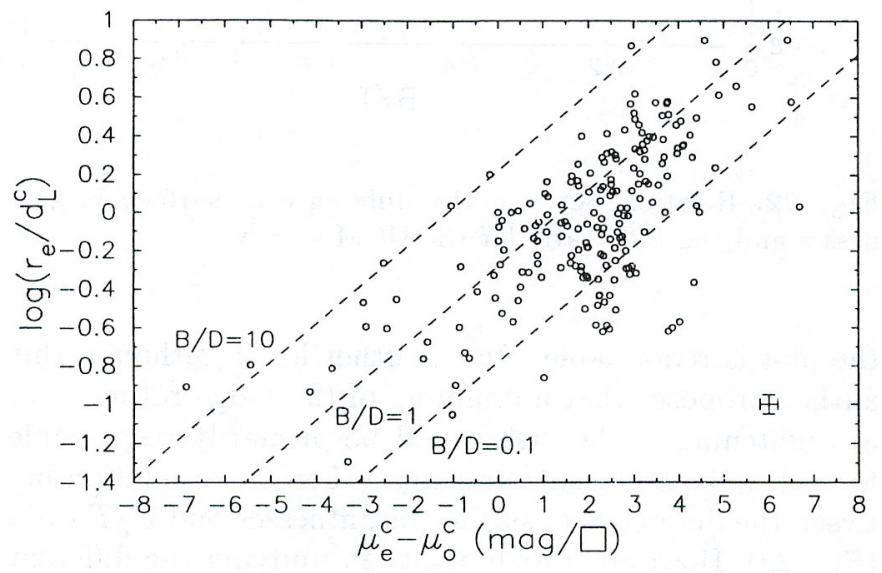

Fig. 24. Dependence between the ratio of scale lengths and the difference of surface brightnesses for the UCM survey. The dashed lines show the positions of constant $B / D$ ratio in sequences of $\log (B / D)= \pm 1$

We have also analysed the dependence between the ratio of scale lengths and the difference between bulge and disk surface brightnesses. The trend, represented in Fig. 24 , is similar to that observed by Kodaira et al. (1986) for a heterogeneous sample of 167 galaxies. This plot corresponds in fact to a distribution of the objects according to their $B / D$ ratio, since from

$$
\frac{B}{D}=3.607\left(\frac{r_{\mathrm{e}}}{d_{\mathrm{L}}}\right)^{2} \operatorname{dex}\left(-0.4\left(\mu_{\mathrm{e}}-\mu_{0}\right)\right)
$$

that is

$$
\log \left(\frac{r_{\mathrm{e}}}{d_{\mathrm{L}}}\right)=0.5 \log \left(\frac{B}{D}\right)-0.279+0.2\left(\mu_{\mathrm{e}}-\mu_{0}\right)
$$

the points with a constant $B / D$ ratio would distribute along straight lines with

$$
\log \left(\frac{r_{\mathrm{e}}}{d_{\mathrm{L}}}\right)=C+0.2\left(\mu_{\mathrm{e}}-\mu_{0}\right)
$$

Therefore, and as can be seen in the figure, most of the UCM objects are below $B / D=1$, as corresponds to a late-type dominated sample, and the region with $B / D$ $>10$ is populated by S0 types.

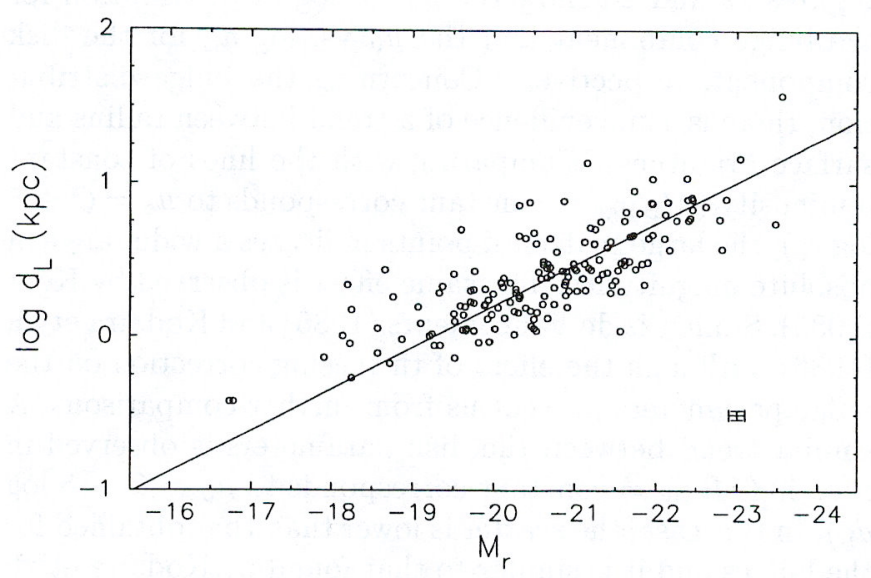

Fig. 25. Correlation between $M_{r}$ and the exponential scale length of the disk, $d_{\mathrm{L}}$, for the UCM survey. A linear least-squares fit to the data is shown

Finally, we have investigated the correlations of the parameters obtained from the $B+D$ decomposition with the absolute magnitude of the galaxies. The only parameter that shows a clear correlation with $M_{r}$ is the disk exponential scale length. This dependence, shown in Fig. 25 , represents a clear influence of disk scales in the total luminosity of galaxies, as expected for disk-dominated systems. A linear least-squares fit to the data leads to the following relation:

$$
\log d_{\mathrm{L}}=(-5.20 \pm 0.29)+(-0.27 \pm 0.01) M_{T}
$$




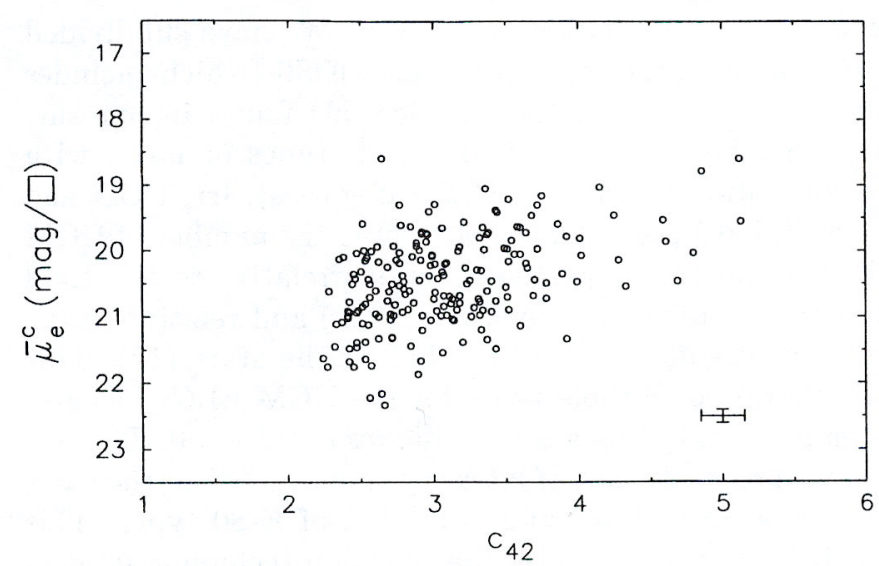

Fig. 26. Mean surface effective brightnesses $\bar{\mu}_{\mathrm{e}}$ as a function of the $c_{42}$ concentration index for the UCM survey

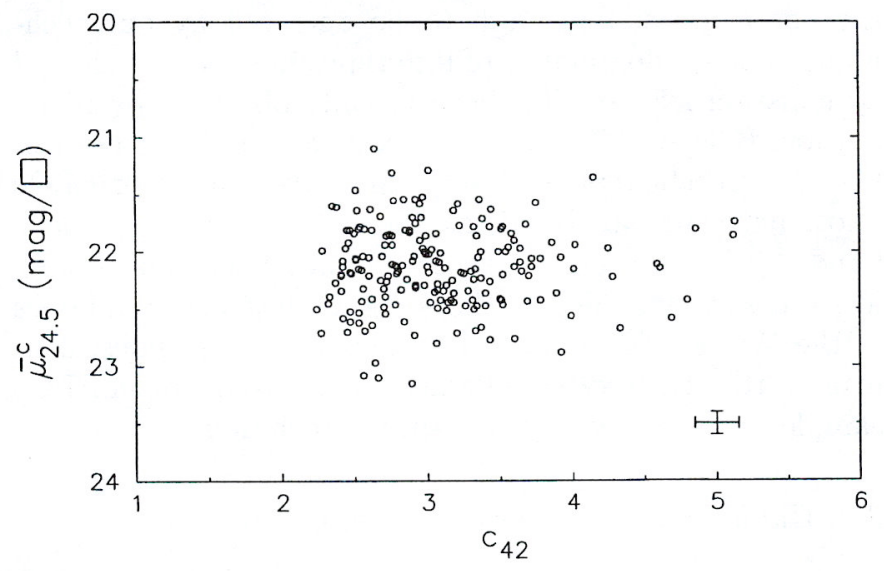

Fig. 27. Mean surface brightness inside the $24.5 \mathrm{mag} / \operatorname{arcsec}^{2}$ isophote, $\bar{\mu}_{24.5}$, as a function of the $c_{42}$ concentration index for the UCM survey

\subsection{Relations concerning mean photometric parameters}

We have studied the relations between mean surface brightnesses and concentration indices. In Fig. 26 a trend is shown in the sense that high $c_{42}$ objects (early-type galaxies) exhibit relatively brighter mean surface effective brightnesses $\bar{\mu}_{\mathrm{e}}$, in agreement with Kent (1985). The same effect is obtained when replacing $c_{42}$ by $c_{31}$. This trend reflects that both parameters represent a good quantitative estimation of the concentration of the light in the galaxies. However, the mean surface brightness inside the 24.5 $\mathrm{mag} / \operatorname{arcsec}^{2}$ isophote, $\bar{\mu}_{24.5}$, is rather independent of the $c_{42}$ or $c_{31}$ indices, as shown in Fig. 27, since the concentration of the light is diluted when averaging over the entire galaxy.

Although $\bar{\mu}_{24.5}$ is insensitive to the Hubble type, Doi et al. (1993) showed its utility as a segregation morphological parameter when combining it with the $c_{\text {in }}(\alpha)$ in-

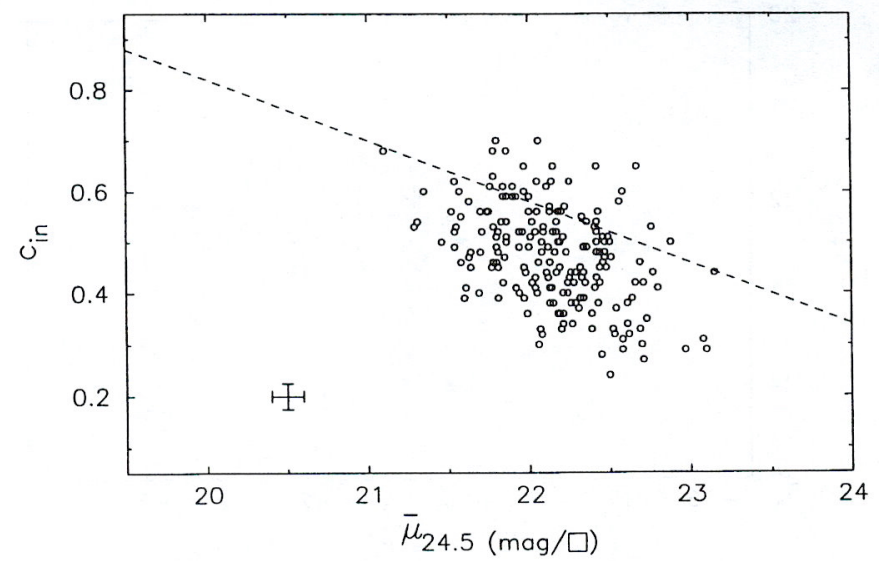

Fig. 28. Positions on the $\left(c_{\text {in }}, \bar{\mu}_{24.5}\right)$ plane of Doi et al. (1993) for UCM objects. The dashed line is the segregation line between early and late-type galaxies proposed by these authors

dex. In fact, the plane defined by these two parameters has been one of the five morphological classifiers used in Paper I. Figure 28 shows the distribution of the UCM sample in this plane. The majority of the UCM objects are placed below the straight line of segregation proposed by Doi et al. (1993), that is, corresponds to Hubble types $\mathrm{Sa}$ or later. The UCM sample is confined within a region of about $2 \mathrm{mag}$ in $\bar{\mu}_{24.5}$ and 0.5 units in $c_{\mathrm{in}}(\alpha)$. All of the objects above the segregation line have a high $B / D$ ratio, corresponding to early types. In particular, all the Sy 1 in our sample are within the region $\bar{\mu}_{24.5}=21.8-$ $22.4 \mathrm{mag} / \operatorname{arcsec}^{2}$ and $c_{\text {in }}(\alpha)=0.6-0.7$, whereas the Sy 2 lay below that straight line and scatter across all the morphologies. This fact confirms the known trend of the Sy 1 nuclei to be hosted in early types galaxies, whereas Sy 2 are usually located in later types. Moreover, all the UCM galaxies with an aspect clearly irregular on the direct image and classified also as Irr according to our fivecriteria method (Paper I), are located in the plot towards the lower right corner, as expected for the latest Hubble types, and the same trend is observed for the BCD candidates.

Finally, in Fig. 29 we have analysed the possible dependence between $\bar{\mu}_{24.5}$ and the absolute magnitude $M_{r}$. The observed independence of $\bar{\mu}_{24.5}$ on the total luminosity agrees with a roughly constant light/area ratio when averaging over the entire galaxy.

\section{Morphological statistics of the objects in the UCM survey}

In this section we present a statistical analysis of the morphological aspects (location of emitting knots, Hubble types, tidal features, etc.) for the UCM objects. 


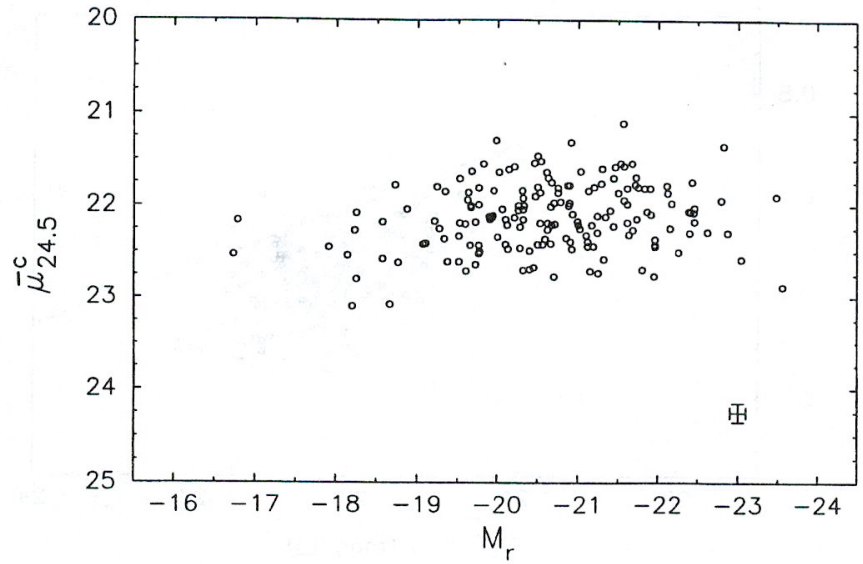

Fig. 29. Mean surface brightness inside the $24.5 \mathrm{mag} / \mathrm{arcsec}^{2}$ isophote, $\bar{\mu}_{24.5}$, as a function of the absolute magnitude $M_{r}$ for the UCM survey

\subsection{Location of emitting knots. Frequency of Hubble types}

We have found that only $22 \%$ of the UCM galaxies $(42 / 194)$ show star formation regions outside the nucleus. In no way this result can be extrapolated to the relative frequency of ELGs with non-nuclear star forming regions in the Local Universe, since our survey, like others, presents complicated selection effects.

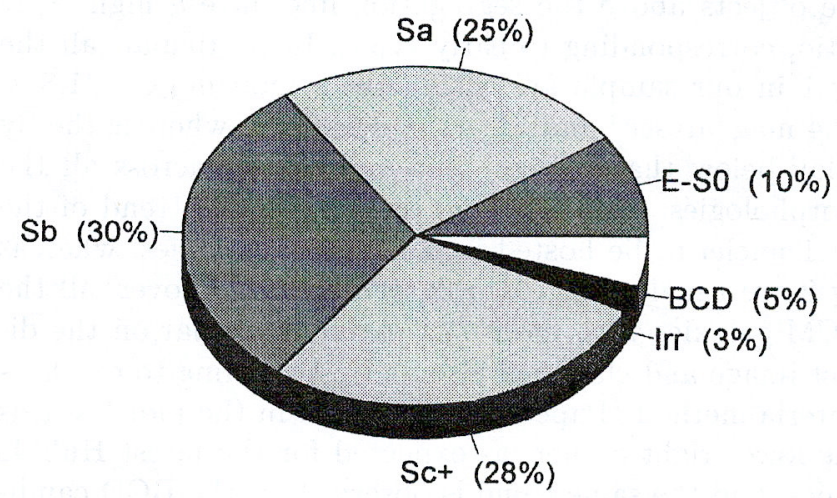

Fig. 30. Diagram of the distribution of Hubble types for the UCM ELGs

Table 2. Hubble types for the UCM objects

\begin{tabular}{cccccccc}
\hline Type & E-S0 & $\mathrm{Sa}$ & $\mathrm{Sb}$ & $\mathrm{Sc}^{+}$ & $\mathrm{Irr}$ & $\mathrm{BCD}$ & Stars \\
\hline$N$ & 19 & 48 & 59 & 54 & 5 & 9 & 18 \\
objects & $9 \%$ & $23 \%$ & $28 \%$ & $25 \%$ & $2 \%$ & $4 \%$ & $8 \%$ \\
only galaxies & $10 \%$ & $25 \%$ & $30 \%$ & $28 \%$ & $3 \%$ & $5 \%$ & - \\
\hline
\end{tabular}

Concerning the morphologies, we have carried out a statistical analysis of the UCM sample and also in compar- ison with other samples of galaxies. We have subdivided the sample in the following types: E-SO (which includes all the UCM S0 and the elliptical $\mathrm{cD}$ found in our survey), $\mathrm{Sa}, \mathrm{Sb}, \mathrm{Sc}^{+}$(that includes $\mathrm{Sc}$ types or later, with the exception of Irr and BCD categories), Irr, BCD and stars. Table 2 presents, for each type, the number of UCM objects and the respective fractions relative to the total number of objects in the survey (212) and relative to the number of galaxies (194), excluding the stars (18). This distribution of Hubble types for the UCM ELGs has also been represented by a sector diagram in Fig. 30. There is a clear predominance of later types, with $66 \%$ of the sample being $\mathrm{Sb}$ or later and only $10 \%$ of E-S0 types. This result is a consequence of the selection technique used in the UCM survey, since the galaxies have been selected by the presence of emission features. Within the latest types, the paucity of Irr galaxies in the UCM survey could come from the fact that, in these systems, the emitting regions are not contrasted enough to be detected by our technique. The predominance of late types in samples selected by emission features has been already observed by many authors (Gisler 1978; and Moss \& Whittle 1993), both in field and in clusters. Although the only sample of ELGs photometrically analysed, as the UCM survey, in the $r$ band, by Bothun et al. (1989) has not been morphologically described, these authors claim that many galaxies in the Wasilewski sample show exponential profiles, confirming that early types galaxies are not frequent in ELGs samples selected by objective-prism technique.

\subsection{Hubble types for special classes of galaxies}

In the UCM subsample of 12 Seyfert galaxies, the five Sy 1 present S0 types whereas the seven Sy 2 show later morphologies (one $\mathrm{Sa}$, three $\mathrm{Sb}$ and three $\mathrm{Sc}$ ). This result confirms the predominance of early types among the Sy 1 and that of later types for Sy 2, as mentioned in the previous section.

The global frequency of barred systems in the ELGs of the UCM survey is $9 \%(18 / 194)$. Considering the Hubble types, $5 \%$ of the barred UCM galaxies are S0, $17 \%$ are $\mathrm{Sa}, 50 \%$ are $\mathrm{Sb}$ and the remaining $28 \%$ are $\mathrm{Sc}^{+}$, showing then a predominance of later types, as in the whole UCM sample. And about the bar/ring-activity connection, in our subsample of 12 Seyfert galaxies, only two cases $(11 \%)$ clearly present an underlying barred structure (UCM 1659+2928 and UCM 0142+2137) and only one (UCM 2329+2500) shows a ring (Gallego et al. 1994).

Since star formation is enhanced by interactions between galaxies, we have investigated the frequency of interacting systems in our survey of ELGs. In our exploration we have detected 11 systems with interacting features. These systems involve 16 UCM ELGs, since the remaining galaxies does not show obvious emission in our objective-prism plates due to either their weakness or their saturation on the plates. In the interacting UCM 
subsample of ELGs, $25 \%$ are S0, $19 \% \mathrm{Sa}, 44 \% \mathrm{Sb}$ and the remaining $12 \%$ are $\mathrm{Sc}^{+}$. Therefore, this subsample is also dominated by late-type galaxies, although there is a relatively high fraction of S0's. This fact is in agreement with the idea proposed by some authors (e.g. van der Bergh $1990)$ in the sense that, at least in some cases, the S0 morphology could have been caused by present encounters or past mergers. About the presence of bars, two UCM interactive galaxies (13\%), the SBb UCM $1537+2506 \mathrm{~N}$ and the SBa UCM $1537+2506 \mathrm{~S}$, are barred. Concerning the occurrence of activity, 2 out of 16 interacting galaxies (13\%) present Seyfert features (the Sy 1 UCM $1701+3131$ and the Sy 2 UCM 1259+2934). Finally, in a global context, the 16 galaxies implicated in interactions represent $8 \%$ of the total UCM ELGs, a fraction lower than the $22 \%$ found in the Wasilewski sample (Bothun et al. 1989), although the problems in the characterization of an interaction only by means of the inspection of the image must be considered.

\subsection{SO galaxies in the UCM ELGS}

Whereas in clusters, a fraction of S0 galaxies could have gas-depleted spirals as progenitors, the origin of field S0 galaxies is uncertain (gas loss by galactic winds, interactions, etc.). We have analysed the S0 nature from a photometric point of view. Following Kent (1985), if S0's were originated from spirals depleted of their gas contents, this would lead to a cessation of star formation and then a dimming in the disks would also be observed. Therefore, an increase in the $B / D$ ratio should be expected, as well as a decrease in the effective radius $a_{\mathrm{e}}$ and the mean surface brightness inside the effective radius $\bar{\mu}_{\mathrm{e}}$. To the contrary to this idea of S to S0 evolution, Kent (1985) finds that $\mu_{0}$ values of S0's are similar to those of spirals, $\mu_{\mathrm{e}}$ values for bulges of S0 are about 2 mag brighter than those of Sc galaxies and that S0's exhibit $\bar{\mu}_{\mathrm{e}}$ not weaker but brighter that those of spirals.

Even taking into account that our S0 galaxies have been selected by the presence of emission features in their spectra and considering the problems in the $B+D$ decomposition for this morphological type, we have studied this problem in the way proposed by Kent (1985). For the subsample of $17 \mathrm{~S} 0$ field galaxies we have found $\mu_{0}$ values about $1.5 \mathrm{mag}$ weaker than the UCM sample mean, but me values for bulges of S0 about 2 mag brighter than the mean, in agreement with Kent (1985). Concerning the length-scales for S0, we find a 20\% decrease in $r_{\mathrm{e}}$ and a $68 \%$ increase in $d_{\mathrm{L}}$ when comparing with the mean values of the whole sample. Globally combined these effects, they cause the expected rise in the $B / D$ ratio when passing from $\mathrm{S}$ to $\mathrm{S} 0$ galaxies. We have also found that the absolute magnitudes for S0 bulges, $M_{\text {Bulge }}$, are $1.6 \mathrm{mag}$ brighter than the mean, whereas for the disks, $M_{\text {Disk }}$, they are $0.3 \mathrm{mag}$ weaker than the mean of the sample. Finally, concerning the mean photometric parameters, we find for
S0's a drop of $13 \%$ in $a_{\mathrm{e}}$ and a brightening of $0.8 \mathrm{mag}$ in $\bar{\mu}_{\mathrm{e}}$ when comparing with the mean values for the UCM sample. In summary, although some of these results (especially the rises in $\mu_{\mathrm{e}}$ and $\bar{\mu}_{\mathrm{e}}$ ) support the ideas of Boroson (1981) and Kent (1985) against a S to S0 evolution in the field, others like a dimming in $\mu_{0}$ for $\mathrm{S} 0$ are opposed to the result found by Kent (1985).

Table 3. Morphological comparison for ELGs between UCM and Gisler samples

\begin{tabular}{lcccc}
\hline Sample & E-S0 & Sa & Sb & $\geq$ Sc \\
\hline Gisler & $12 \%$ & $10 \%$ & $28 \%$ & $50 \%$ \\
UCM & $10 \%$ & $25 \%$ & $30 \%$ & $35 \%$ \\
\hline
\end{tabular}

\subsection{Comparison with Gisler sample}

We compare here the UCM distribution of morphologies with that from previous studies. Since a morphological classification of galaxies selected by an objective-prism technique has been never undertaken so far, we have only compared our results with a sample of emission-line galaxies, selected by different methods. This is the sample of Gisler (1978), with 1316 galaxies (799 with emission). For comparison purposes we have binned Gisler morphological groups in our types E-S0, Sa, Sb and $\mathrm{Sc}^{+}$(Sc or later). Table 3 shows that whereas the fractions of early galaxies (E-S0) and Sb type are very similar in both samples, we have a higher detection of $\mathrm{Sa}$ and a lower fraction of latetype $\left(\mathrm{Sc}^{+}\right)$galaxies. Our excess in Sa detections could be due to the frequent presence of nuclear emission in our sample. And, concerning the excess of $\mathrm{Sc}^{+}$galaxies in the Gisler sample, it could come from the inclusion by this author of a not specified number of galaxies with multiple or unclassified morphologies. These difficulties in the morphological comparisons reveal the necessity of Hubble classifications for recent objective-prism explorations of ELGs as Wasilewski or Michigan surveys.

\section{Influence of the environment in the presence and nature of ELGs}

Since one of the objective-prism plates in the UCM survey covers the Coma cluster, we have analyze whether there is some influence of the environment in the frequency and nature of ELGs when comparing our samples in the field and in the cluster medium. This comparison must be considered as indicative since only a cluster is concerned in our observations. 


\subsection{Frequency and Hubble types of UCM ELGs in the Coma cluster}

A previous analysis for the UCM survey (Zamorano et al. 1996) has shown that the fraction of detected ELGs relative to the total number of Zwicky galaxies in the same area of the sky goes down from $15 \%$ to $7 \%$ when comparing the field to the Coma cluster. This decrease in the fraction of ELGs in a dense medium, has been previously observed e.g. by Gisler (1978), Dressler et al. (1985) and Salzer et al. (1988), and it is related to the morphologydensity relation (Dressler 1980): in a denser environment, a lower quantity of gas available corresponds to a higher predominance of early-type (E-S0) galaxies and, therefore, to a reduced presence of star-forming systems, dominated by late-type galaxies.

In Table 4 we present the morphological distribution for UCM galaxies in the field (157) and in the Coma cluster (37). Although in both environments the sample is dominated by late-type galaxies, when passing from the field to the cluster we observe drastic reductions in the fraction of early-types (E-S0) ELGs and late-type spirals $\left(\mathrm{Sc}^{+}\right)$, although there is an increase in the Irr class. On the other hand, the frequency of Sa remains almost the same, and there is a significative increase in the fraction of $\mathrm{Sb}$ galaxies. Since this analysis has never been undertaken in other surveys of ELGs selected by an objective-prism technique, we only have been able to make a comparison with some samples of galaxies selected by other criteria. So, the same effects, excluding the reduction in the latetype galaxies, were observed by Gisler (1978). However, Moss \& Whittle (1993) found no difference in the fraction of early-types ELGs when comparing the field with a cluster environment, although they found the same effect in the reduction of late-type spirals.

\subsection{Interpretation of the observed results in Coma}

Although the distribution of morphological types in the Coma cluster has been investigated by several authors (e.g. Doi et al. 1995), the interpretation of our results is not straightforward since it should not be forgotten that our subsample in Coma does not correspond to a general sample of normal galaxies, but of galaxies selected by the presence of emission features in their objective-prism spectra. Thus, in our subsample of Coma galaxies, we are biased to star forming systems (i.e. late-type galaxies) that, as proposed by e.g. Bothun et al. (1984), could afterwards evolve to early-type galaxies due to a sweeping of their gas when falling to the cluster center. Concerning the excess in the Irr type in Coma, although Gallagher \& Hunter (1989) propose a lower star formation efficiency in the cluster Irr compared to field Irr, we find that 4 out 5 (80\%) of the irregular galaxies found in the UCM survey belong to the Coma cluster. The high fractions of Sa-Sb types observed in our survey could be explained by the excess of red color in the central parts of the disk galaxies observed by Gavazzi et al. (1990). Finally, concerning the presence of interactions, we find a reduction from $9 \%$ to $5 \%$ when comparing the fraction of galaxies with signs of interactions in the field to that in the Coma cluster. This could suggest that in the regions of low local galaxy density, the smaller relative velocity in the encounters between galaxies could favour the interacting systems. Unfortunately, since the question concerning whether the star formation rate is lower (Gisler 1978) or higher (Maia et al. 1994) in denser environments compared to the field has not been answered yet, and considering the instrumental effect of the different selection techniques (Moss \& Whittle 1993), any comparison must be taken with caution.

\section{Summary}

We have carried out an extensive Thuan \& Gunn $r$ CCD imaging of the Universidad Complutense de Madrid (UCM) sample of emission-line galaxies (ELGs), which have been selected from the presence of $\mathrm{H} \alpha$ emission in low resolution objective-prism plates. In Paper I (Vitores et al. 1996) we presented the observations and basic reductions for the set of 212 objects from the UCM survey. Also, a set of photometric parameters has been calculated to obtain, by using simultaneously five criteria, a morphological classification of the objects. In this paper we have analysed the statistical properties of the sample and their interpretations.

For the first time in a sample of ELGs, a complete analysis of the photometric data and morphological types has been carried out. For the whole UCM sample, we present histograms for all the computed parameters: magnitudes, sizes, $B+D$ decomposition values, mean photometric radii and surface brightnesses, and concentration indices. Some correlations between these photometric parameters have also been analysed.

The statistical analysis of the obtained results has shown that, photometrically, our survey selects objects fainter (in apparent magnitudes) than those of color surveys and confirms that objective-prism surveys carried out in the blue and in the red bands are complementary to each other. In absolute values, our survey also confirms the advantage of emission-line surveys in detecting objects intrisically fainter than those found from other selection techniques. The UCM survey attains median values for the whole sample of $M_{r}=-20.6$ and $D_{24}=19.4 \mathrm{kpc}$. Morphologically, the sample comprises a very heterogeneous population of galaxies, although it is dominated by late-type galaxies ( $66 \% \mathrm{Sb}$ or later), as it is confirmed by means of several criteria. We propose a set of 9 candidates to $\mathrm{BCD}$ (blue compact dwarf) galaxies, five of them previously unknown. We have found that roughly $10 \%$ of the ELGs of the sample exhibit typical parameters of S0 types and, in the subsample of the $16 \mathrm{UCM}$ interacting galaxies, $25 \%$ are classified as S0's. 
Table 4. Morphological comparison for UCM galaxies in the field and in the Coma cluster. Between parenthesis is presented the total number of galaxies for each category

\begin{tabular}{ccccccc}
\hline Subsample & E-S0 & Sa & Sb & Sc $^{+}$ & Irr & BCD \\
\hline Field & $11 \%(18)$ & $24 \%(38)$ & $28 \%(44)$ & $31 \%(48)$ & $1 \%(1)$ & $5 \%(8)$ \\
Cluster & $3 \%(1)$ & $27 \%(10)$ & $41 \%(15)$ & $16 \%(6)$ & $11 \%(4)$ & $3 \%(1)$ \\
\hline
\end{tabular}

Finally, our study of the rich Coma cluster by means of the objective-prism technique allows the dectection of a population of late Hubble types. The photometric analysis has found that $71 \%$ of the UCM ELGs in the Coma cluster are classified as $\mathrm{Sb}$ type or later.

Acknowledgements. The authors are indebted to J. Gorgas for his continuous cooperation, valuable ideas and careful reading of the manuscript. This work was supported in part by the Spanish "Programa Sectorial de Promoción General del Conocimiento" under grant PB93-456.

\section{References}

Boroson T., 1981, ApJS 46, 177

Bothun G.D., Schommer R.A., Sullivan W.T. III, 1984, AJ 89, 466

Bothun G.D., Halpern J.P., Lonsdale C.J., Impey C., Schmitz M., 1989, ApJS 70, 271

Comte G., Augarde R., Chalabaev A., Kunth D., Maehara H., 1994, A\&A 285, 1

de Vaucouleurs G., 1977, Evolution of Galaxies and Stellar Populations. In: Larson R., \& Tynsley B. (eds.). Yale Univ. Observatory, New Haven, p. 43

Doi M., Fukugita M., Okamura S., 1993, MNRAS 264, 832

Doi M., Fukugita M., Okamura S., Turner E.L., 1995, AJ 109, 1490

Dressler A., 1980, ApJ 236, 351

Dressler A., Thompson I.B., Shectman S.A., 1985, ApJ 288, 481

Freeman K.C., 1970, ApJ 160, 811

Gallagher III J.S., Hunter D.A., 1989, AJ 98, 806

Gallego J., Zamorano J., Rego M., Vitores A.G., 1994, A\&A 290,705

Gallego J., Zamorano J., Rego M., Alonso O., Vitores A.G., 1996, A\&AS (submitted)

Gavazzi G., Garilli B., Boselli A., 1990, A\&AS 83, 399

Gisler G.R., 1978, MNRAS 183, 633

Izotov Y.I., Lipovetskii V.A., Guseva N.G., et al., 1993, private communication to J. Gallego in Panchromatic View of Galaxies, Kiel
Kent S.M., 1984, ApJS 56, 105

Kent S.M., 1985, ApJS 59, 115

Kodaira K., Watanabe M., Okamura S., 1986, ApJS 62, 703

MacKenty J.W., 1990, ApJS 72, 231

Maia M.A., Pastoriza M.G., Bica E., Dottori H., 1994, ApJS 93,425

Mazzarella J.M., Balzano V.A., 1986, ApJS 62, 751

Moss C., Whittle M., 1993, ApJ 407, L17

Okamura S., Doi M., Fukugita M., et al., 1994, Automatic morphological classification of galaxies. In: MacGillivray H.T., Thomson E.B., Lasker B.M., Reid I.N., Malin D.F., West R.M., Lorenz H. (eds.) Proceed. IAU Symp. 161, Astronomy from Wide-Field Imaging. Kluwer Academic Publ., Holland, p. 243

Paturel G., Fouqué P., Bottinelli L., Gouguengheim L., 1989, Catalogue of Principal Galaxies (PGC). Observatoires de Lyon et Paris-Meudon. Lyon (France)

Romanishin W., Strom K.M., Strom S.E., 1983, ApJS 53, 105

Salzer J.J., Aldering G.S., Bothun G.D., Mazzarella J.M., Lonsdale C.J., 1988, AJ 96, 1511

Salzer J.J., MacAlpine G.M., Boroson T.A., 1989, ApJS 70, 479

Simien F., 1989, Photometric decomposition of galaxies. In: Corwin H.G., \& Bottinelli L. (eds.) The World of Galaxies. Springer-Verlag, New York, p. 293

Simien F., de Vaucouleurs G., 1986, ApJ 302, 564

Takase B., Miyauchi-Isobe N., 1993, Publ. Natl. Astron. Obs. Japan Vol. 3, 169

Thuan T.X., Gunn J.E., 1976, PASP 88, 543

van der Bergh S., 1990, ApJ 348, 57

van der Kruit P.C., 1987, A\&A 173, 59

van der Kruit P.C., 1989, Photometry of disks in galaxies. In: Corwin H.G., \& Bottinelli L. (eds.) The World of Galaxies. Springer-Verlag, New York, p. 256

Vitores A.G., Zamorano J., Rego M., Alonso O., Gallego J., 1996, A\&AS 118, 7 (Paper I)

Wasilewski A.J., 1983, ApJ 272, 68

Zamorano J., Rego M., Gallego J., Vitores A.G., GonzálezRiestra R., Rodríguez-Caderot G., 1994, ApJS 95, 387

Zamorano J., Gallego J., Rego M., Vitores A.G., Alonso O., 1996, ApJS (in press) 